\title{
Statistical Performance of MUSIC-Like Algorithms in Resolving Noncircular Sources
}

\author{
Habti Abeida and Jean-Pierre Delmas, Senior Member, IEEE
}

\begin{abstract}
This paper addresses the resolution of the conventional and noncircular MUSIC algorithms for arbitrary circular and noncircular second-order distributions of two uncorrelated closely spaced transmitters observed by an arbitrary array. An explicit closed-form expression of the mean null spectrum of the conventional and noncircular MUSIC algorithms is derived using an analysis based on perturbations of the noise projector instead of those of the eigenvectors. Based on these results, theoretical and approximate interpretable closed-form expressions of the threshold array signal-to-noise ratios (ASNR) at which these two algorithms are able to resolve two closely spaced transmitters along the Cox and the Sharman and Durrani criteria are given. It is proved that the threshold ASNRs given by the conventional MUSIC algorithm do not depend on the distribution of the sources including their noncircularity, in contrast to the noncircular MUSIC algorithm for which they are very sensitive to the noncircularity phase separation of the sources. This threshold ASNR given by the noncircular MUSIC algorithm is proven to be comfortably lower than that given by the conventional MUSIC algorithm except for weak phase separations of the sources for which the resolving powers of these two algorithms are very close. Finally, these results are analyzed through several illustrations and Monte Carlo simulations.
\end{abstract}

Index Terms-Circular and noncircular signal, direction of arrival (DOA), MUSIC algorithm, threshold of resolution.

\section{INTRODUCTION}

D EDUCING array resolution limits is a very old problem that has been studied extensively in the literature, first in astronomy and subsequently in signal processing. Based on the classical beamformer, different resolution criteria have been defined from the main lobe of the array spectrum as the celebrated Rayleigh resolutions such as the half power beamwidth or the null to null beamwidth [1] that depends solely on the antenna geometry.

Then for specific so-called high-resolution algorithms based on the search for two local minima of null sample spectra such as different MUSIC-like algorithms, two main criteria based on the mean null spectrum have been defined. For the first, introduced by Cox [2], two sources are resolved if the midpoint mean null spectrum is greater than the mean null spectrum in the two true source DOAs. This criterion was first studied by Kaveh and Barabell [3], [4] in the resolution analysis of the conventional MUSIC and Min-Norm algorithms for two uncorrelated

Manuscript received July 16, 2007; revised March 13, 2008. Published August 13, 2008 (projected). The associate editor coordinating the review of this manuscript and approving it for publication was Dr. Sergiy A. Vorobyov.

The authors are with the Institut TELECOM SudParis, Département CITI, CNRS UMR 5157, 91011 Evry Cedex, France (e-mails: abeida3@yahoo.fr; jean-pierre.delmas@it-sudparis.eu).

Digital Object Identifier 10.1109/TSP.2008.924143 equal-power sources and a uniform linear array (ULA). This analysis has been extended to more general classes of situations, e.g., for two correlated or coherent equal-power sources with the smoothed MUSIC algorithm [5], then for two unequipowered sources impinging on an arbitrary array with the conventional and beamspace MUSIC algorithm [6]. A subsequent paper by Zhou et al. [7] developed a resolution measure based on the mean zero spectrum and compared their results to Kaveh and Barabell's work. For the second one, introduced by Sharman and Durrani [8] and then studied by Forster and Villier [9], [10] in the context of the conventional MUSIC and Min-Norm algorithms, for two uncorrelated equal-power sources and a ULA, two sources are resolved if the second derivative of the mean spectrum at the midpoint is negative.

Moreover, several authors have considered (e.g., [11]-[13]) the probability of resolution or an approximation of it based on the Cox criterion applied to the sample null spectrum to circumvent the possible misleading results given by these two criteria. Finally array resolution limits has been studied independently of any algorithm from different points of view. Based on order detection using information theoretic criteria such that the minimum description length (MDL) and the Akaike's information (AIC) criteria, the probability of underestimating or overestimating the number of sources for the case of two closely spaced sources has been analyzed in [4]. Resolution criteria have been defined from the Cramér-Rao bound (CRB) [14] as the source separation that equals its own CRB, then applied to discrete sources in [15], and from the generalized likelihood ratio test [16] through constraints on the probabilities of false alarm and of detection.

We note that all these studies have been obtained under a circular Gaussian distribution of signals. The aim of this paper is to extend some of these previous results under arbitrary second-order distributions, with a particular attention to noncircular signals often used in digital communications. More precisely, we consider the two resolution criteria based on mean null spectra associated with the conventional MUSIC algorithm and with a MUSIC-like (denoted noncircular MUSIC) algorithm introduced and studied in [17] which is an extension of a root MUSIC-like algorithm devised in [18] to an arbitrary array that benefits from the second-order noncircularity of the sources.

The paper is organized as follows. The array signal model and the statement of the problem are given in Section II. Using an analysis based on perturbations of the noise projector instead of those of the eigenvectors, an explicit closed-form expression of the mean null spectrum of conventional MUSIC algorithm that does not depend on the distribution including the noncircularity of the sources is derived in Section III. Consequently, the associ- 
ated expressions (e.g., [3, rel. (35)] and [9, rel. (18)]) of the resolution thresholds obtained under the two foregoing criteria for circular Gaussian sources extend to arbitrary distributions and arbitrary arrays. This analysis is applied again in Section IV, to derive closed-form expressions of the null spectrum of the noncircular MUSIC algorithm. Then, closed-form expressions of the resolution thresholds are deduced under the two foregoing criteria for arbitrary arrays. These expressions show the crucial role played by the noncircularity phase separation of the sources. They confirm that the noncircular MUSIC algorithm largely outperforms the conventional MUSIC algorithm from the resolution point of view for large noncircularity phase separation, in contrast to weak noncircularity phase separation for which the threshold ASNRs given for the noncircular and conventional MUSIC algorithms are similar. Finally, numerical illustrations and Monte Carlo simulations are given in Section V with particular attention paid to the noncircularity phase separation and the main results are summarized in a conclusion.

The following notations are used throughout the paper. Matrices and vectors are represented by bold upper case and bold lower case characters, respectively. Vectors are by default in column orientation, while $T, H, *$, and \# stand for transpose, conjugate transpose, conjugate, and Moore-Penrose inverse, respectively. $\mathrm{E}(),. \operatorname{Tr}(),. \operatorname{Det}(.)\|.\|_{\mathrm{Fro}}, \Re($.$) and \Im($. are the expectation, trace, determinant, Frobenius norm, real and imaginary part operators respectively. $\mathbf{I}_{K}$ is the $K$-order identity matrix, $\mathbf{e}_{K, k}$ denotes the $k$-unit vector in $\mathbb{R}^{K}$, and $\mathbf{J}$ is the $2 M \times 2 M$ partitioned matrix $\left(\begin{array}{cc}\mathbf{O} & \mathbf{I}_{M} \\ \mathbf{I}_{M} & \mathbf{O}\end{array}\right) \cdot \operatorname{vec}(\cdot)$ is the "vectorization" operator that turns a matrix into a vector by stacking the columns of the matrix one below another which is used in conjunction with the Kronecker product $\mathbf{A} \otimes \mathbf{B}$ as the block matrix whose $(i, j)$ block element is $a_{i, j} \mathbf{B}$ and with the vec-permutation matrix $\mathbf{K}$ which transforms $\operatorname{vec}(\mathbf{C})$ to $\operatorname{vec}\left(\mathbf{C}^{T}\right)$ for any square matrix $\mathbf{C}$.

\section{Statement of THE Problem}

Let an arbitrary array of $M$ sensors receive the signals transmitted by $K$ equal-power narrowband independent sources of power $\sigma_{x_{k}}^{2}$. The observation vectors are modeled as

$$
\mathbf{y}_{t}=\mathbf{A} \mathbf{x}_{t}+\mathbf{n}_{t}, \quad t=1, \ldots, T
$$

where $\left(\mathbf{y}_{t}\right)_{t=1, \ldots, T}$ are independent and identically distributed. $\mathbf{A}=\left[\mathbf{a}_{1}, \ldots, \mathbf{a}_{K}\right]$ is the steering matrix where each vector $\mathbf{a}_{k}=$ $\mathbf{a}\left(\theta_{k}\right)$ is parameterized by the real scalar parameter $\theta_{k}$ according to the parametrization introduced in [6] where $\left\|\mathbf{a}_{k}\right\|^{2}=M$. $\mathbf{x}_{t}=\left(x_{t, 1}, \ldots, x_{t, K}\right)^{T}$ and $\mathbf{n}_{t}$ model signals transmitted by sources and additive measurement noise, respectively. $\mathbf{x}_{t}$ and $\mathbf{n}_{t}$ are independent, zero-mean, $\mathbf{n}_{t}$ is assumed to be Gaussian complex circular, spatially uncorrelated with $\mathrm{E}\left(\mathbf{n}_{t} \mathbf{n}_{t}^{H}\right)=\sigma_{n}^{2} \mathbf{I}_{M}$; while $\mathbf{x}_{t}$ is complex noncircular not necessarily Gaussian with covariance matrices $\mathbf{R}_{x} \stackrel{\text { def }}{=} \mathrm{E}\left(\mathbf{x}_{t} \mathbf{x}_{t}^{H}\right)$ and $\mathbf{R}_{x}^{\prime} \stackrel{\text { def }}{=} \mathrm{E}\left(\mathbf{x}_{t} \mathbf{x}_{t}^{T}\right) \neq$ O. Consequently, this leads to the following two covariance matrices of $\mathbf{y}_{t}$ that contain information about $\left(\theta_{1}, \ldots, \theta_{K}\right)$ :

$$
\mathbf{R}_{y}=\mathbf{A} \mathbf{R}_{x} \mathbf{A}^{H}+\sigma_{n}^{2} \mathbf{I}_{M} \stackrel{\text { def }}{=} \mathbf{S}+\sigma_{n}^{2} \mathbf{I}_{M}
$$

and

$$
\mathbf{R}_{y}^{\prime}=\mathbf{A R}_{x}^{\prime} \mathbf{A}^{T} \neq \mathbf{O} .
$$

These covariance matrices are classically estimated by $\mathbf{R}_{y, T}=(1 / T) \sum_{t=1}^{T} \mathbf{y}_{t} \mathbf{y}_{t}^{H}$ and $\mathbf{R}_{y, T}^{\prime}=(1 / T) \sum_{t=1}^{T} \mathbf{y}_{t} \mathbf{y}_{t}^{T}$, respectively.

For performance analysis, we suppose that the signal waveforms $\left(x_{k, t}\right)_{k=1, \ldots, K}$ have finite fourth-order moments $\kappa_{k} \stackrel{\text { def }}{=}$ $\operatorname{Cum}\left(x_{t, k}, x_{t, k}^{*}, x_{t, k}, x_{t, k}^{*}\right)$. The noncircularity rate $\rho_{k}$ of the $k$ th source is defined by $\mathrm{E}\left(x_{t, k}^{2}\right)=\rho_{k} e^{2 i \phi_{k}} \mathrm{E}\left|x_{t, k}^{2}\right|=\rho_{k} e^{2 i \phi_{k}} \sigma_{x_{k}}^{2}$ where $\phi_{k}$ is its noncircularity phase. Note that $\rho_{k}=1$ in the particular case of rectilinear signals with phase $\phi_{k}$.

The problem addressed in this paper is to derive in these conditions, resolution threshold expressions associated with the conventional and noncircular MUSIC algorithms. The DOA estimated by the conventional MUSIC algorithm are given by the $K$ smallest minima of the following so-called null spectrum $g_{T}^{\mathrm{Alg}_{\mathrm{C}}}(\theta)[19]:$

$$
\widehat{\theta}_{k, T}^{\mathrm{Alg}_{\mathrm{C}}}=\arg \min _{\theta} g_{T}^{\operatorname{Alg}_{\mathrm{C}}}(\theta)
$$

with

$$
g_{T}^{\operatorname{Alg}_{\mathrm{C}}}(\theta) \stackrel{\text { def }}{=} \mathbf{a}^{H}(\theta) \mathbf{\Pi}_{T} \mathbf{a}(\theta)
$$

where $\Pi_{T}$ denotes the projector matrix associated with the noise subspace of $\mathbf{R}_{y, T}$. Then, for the noncircular MUSIC algorithms devised for rectilinear signals, ${ }^{1}$ the estimated DOA are given by the $K$ smallest minima of the following so-called spectrum $g_{T}^{\mathrm{Alg}}(\theta)$ :

$$
\widehat{\theta}_{k, T}^{\mathrm{Alg}_{\mathrm{NC}}}=\arg \min _{\theta} g_{T}^{\mathrm{Alg}_{\mathrm{NC}}}(\theta)
$$

with [17]

$$
g_{T}^{\mathrm{Alg}_{\mathrm{NC}}}(\theta) \stackrel{\text { def }}{=}\left(\mathbf{a}^{H}(\theta) \boldsymbol{\Pi}_{1, T} \mathbf{a}(\theta)\right)^{2}-\left|\mathbf{a}^{T}(\theta) \boldsymbol{\Pi}_{2, T}^{*} \mathbf{a}(\theta)\right|^{2}
$$

where $\Pi_{1, T}$ and $\Pi_{2, T}$ are Hermitian and complex symmetric respectively, given by the projector matrix

$$
\tilde{\Pi}_{T}=\left(\begin{array}{ll}
\Pi_{1, T} & \Pi_{2, T} \\
\Pi_{2, T}^{*} & \Pi_{1, T}^{*}
\end{array}\right)
$$

associated with the noise subspace of $\mathbf{R}_{\tilde{y}, T} \stackrel{\text { def }}{=}$ $(1 / T) \sum_{t=1}^{T} \tilde{\mathbf{y}}_{t} \tilde{\mathbf{y}}_{t}^{H} \quad$ with $\quad \tilde{\mathbf{y}}_{t}$ the extended observation $\left(\begin{array}{l}\mathbf{y}_{t} \\ \mathbf{y}_{t}^{*}\end{array}\right)$ for which

$$
\mathbf{R}_{\tilde{y}} \stackrel{\text { def }}{=} \mathrm{E}\left(\tilde{\mathbf{y}}_{t} \tilde{\mathbf{y}}_{t}^{H}\right)=\tilde{\mathbf{A}} \mathbf{R}_{\tilde{x}} \tilde{\mathbf{A}}^{H}+\sigma_{n}^{2} \mathbf{I}_{2 M} \stackrel{\text { def }}{=} \tilde{\mathbf{S}}+\sigma_{n}^{2} \mathbf{I}_{2 M}
$$

with $\tilde{\mathbf{A}} \stackrel{\text { def }}{=}\left(\begin{array}{cc}\mathbf{A} & \mathbf{O} \\ \mathbf{O} & \mathbf{A}^{*}\end{array}\right)$ and $\mathbf{R}_{\tilde{x}} \stackrel{\text { def }}{=}\left(\begin{array}{cc}\mathbf{R}_{x} & \mathbf{R}_{x}^{\prime} \\ \mathbf{R}_{x}^{\prime *} & \mathbf{R}_{x}^{*}\end{array}\right)$.

\section{Resolving Power of Conventional MUSIC}

Based upon the partially substantiated assumption [3] that the standard deviation $\sqrt{\operatorname{Var}\left[g_{T}^{\operatorname{Alg}_{\mathrm{C}}}(\theta)\right]}$ of the sample null spectrum associated with the conventional MUSIC and Min-Norm algorithms is small compared to its mean value $\mathrm{E}\left[g_{T}^{\mathrm{Alg}_{\mathrm{C}}}(\theta)\right]$ in the

${ }^{1}$ Noncircular with unit rate of noncircularity, i.e., $\rho_{k}=1$. 
vicinity of the emitters, the mean value of the sample null spectrum can be reasonably taken as representative of the ensemble of sample null spectra. We note that the conditions for the validity of this foregoing assumption has been specified in [11], for which it has been proved for the conventional MUSIC algorithm

$$
\operatorname{Var}\left(g_{T}^{\operatorname{Alg}_{\mathrm{C}}}(\theta)\right) \approx \frac{1}{M-K}\left(\mathrm{E}^{2}\left[g_{T}^{\mathrm{Alg}_{\mathrm{C}}}(\theta)\right]-\left[g^{\operatorname{Alg}_{\mathrm{C}}}(\theta)\right]^{2}\right)
$$

in the vicinity of the emitters, for a large number $T$ of snapshots, a fixed number $M$ of sensors ${ }^{2}$ and for arbitrary SNR. Consequently, under the conditions $M \gg 1$ and $T \gg M$

$$
\sqrt{\operatorname{Var}\left[g_{T}^{\mathrm{Alg}_{\mathrm{C}}}(\theta)\right]} \ll \mathrm{E}\left[g_{T}^{\mathrm{Alg}_{\mathrm{C}}}(\theta)\right]
$$

is validated in the vicinity of the emitters. We suppose that (3.2) is valid for the noncircular algorithm as well and based on this assumption, we use the Cox [2] and the Sharman and Durrani criteria [8] for which in the case $K=2$, two closely spaced equal-power sources are resolved if the following respective conditions are satisfied:

$$
\begin{aligned}
\mathrm{E}\left[g_{T}^{\mathrm{Alg}}\left(\theta_{1}\right)\right]=\mathrm{E}\left[g_{T}^{\mathrm{Alg}}\left(\theta_{2}\right)\right] & \leq \mathrm{E}\left[g_{T}^{\mathrm{Alg}}\left(\theta_{m}\right)\right] \\
\frac{d^{2} \mathrm{E}\left[g_{T}^{\mathrm{Alg}}(\theta)\right]}{d \theta^{2}} \quad \mid \theta=\theta_{m} & \leq 0
\end{aligned}
$$

where $\theta_{m} \stackrel{\text { def }}{=}\left(\theta_{1}+\theta_{2}\right) / 2$. Approximations to the resolution threshold are deduced from equalities in (3.3) and (3.4). Consequently, the key point to derive these resolution thresholds depends on the expectation of the random variable $g_{T}^{\mathrm{Alg}_{\mathrm{C}}}(\theta)$. To obtain this expectation, we resort to an analysis based on perturbations of the noise projector [19] instead of those of the eigenvectors (e.g., [3] and [6]). Therefore, we consider the following second-order expansion of $\delta \boldsymbol{\Pi}_{T} \stackrel{\text { def }}{=} \Pi_{T}-\boldsymbol{\Pi}$ w.r.t. $\delta \mathbf{R}_{y, T} \stackrel{\text { def }}{=}$ $\mathbf{R}_{y, T}-\mathbf{R}_{y}$ proved in [19]:

$$
\begin{aligned}
\delta \boldsymbol{\Pi}_{T}= & -\left(\boldsymbol{\Pi} \delta \mathbf{R}_{y, T} \mathbf{S}^{\#}+\mathbf{S}^{\#} \delta \mathbf{R}_{y, T} \boldsymbol{\Pi}\right) \\
& +\mathbf{S}^{\#}\left(\delta \mathbf{R}_{y, T} \boldsymbol{\Pi} \delta \mathbf{R}_{y, T}\right) \mathbf{S}^{\#}-\boldsymbol{\Pi}\left(\delta \mathbf{R}_{y, T} \mathbf{S}^{\# 2} \delta \mathbf{R}_{y, T}\right) \boldsymbol{\Pi} \\
& +\mathbf{S}^{\#}\left(\delta \mathbf{R}_{y, T} \mathbf{S}^{\#} \delta \mathbf{R}_{y, T}\right) \boldsymbol{\Pi}+\boldsymbol{\Pi}\left(\delta \mathbf{R}_{y, T} \mathbf{S}^{\#} \delta \mathbf{R}_{y, T}\right) \mathbf{S}^{\#} \\
& -\mathbf{S}^{\# 2}\left(\delta \mathbf{R}_{y, T} \boldsymbol{\Pi} \delta \mathbf{R}_{y, T}\right) \boldsymbol{\Pi}-\boldsymbol{\Pi}\left(\delta \mathbf{R}_{y, T} \boldsymbol{\Pi} \delta \mathbf{R}_{y, T}\right) \mathbf{S}^{\# 2} \\
& +o\left(\delta \mathbf{R}_{y, T}^{2}\right) .
\end{aligned}
$$

To proceed, we need the expression of $\mathrm{E}\left(\delta \mathbf{R}_{y, T} \mathbf{B} \delta \mathbf{R}_{y, T}\right)$ for arbitrary $M \times M$ matrices $\mathbf{B}$ which is given by the following lemma proved in Appendix A.

Lemma 1: For $K$ independent arbitrary noncircular, possibly non-Gaussian sources, we have

$$
\begin{aligned}
\mathrm{E}\left(\delta \mathbf{R}_{y, T} \mathbf{B} \delta \mathbf{R}_{y, T}\right)=\frac{1}{T}( & \operatorname{Tr}\left(\mathbf{B} \mathbf{R}_{y}\right) \mathbf{R}_{y}+\mathbf{R}_{y}^{\prime} \mathbf{B}^{T} \mathbf{R}_{y}^{\prime *} \\
& \left.+\sum_{k=1}^{K} \kappa_{k} \mathbf{a}_{k} \mathbf{a}_{k}^{H} \mathbf{B} \mathbf{a}_{k} \mathbf{a}_{k}^{H}\right)+o\left(\frac{1}{T}\right)
\end{aligned}
$$

${ }^{2}$ We note that (3.1) is derived from an expansion in $1 / T$ for $M$ fixed, and consequently is only valid for $T \gg M$. Consequently, our analysis is not valid for small sample size scenarios studied in [20] for which $T \sim M$. The domain of validity of our approach will be considered in the illustrative examples Section. that allows us to prove in Appendix B from (3.5) that

$$
\mathrm{E}\left(\delta \boldsymbol{\Pi}_{T}\right)=\frac{1}{T}(\operatorname{Tr}(\mathbf{\Pi}) \mathbf{U}-\operatorname{Tr}(\mathbf{U}) \boldsymbol{\Pi})+o\left(\frac{1}{T}\right)
$$

with $\mathbf{U} \stackrel{\text { def }}{=} \sigma_{n}^{2} \mathbf{S}^{\#} \mathbf{R}_{y} \mathbf{S}^{\#}$. Consequently, we have for $K$ sources

$$
\begin{aligned}
\mathrm{E}\left(g_{T}^{\mathrm{Alg}_{\mathrm{C}}}(\theta)\right)=g^{\mathrm{Alg}_{\mathrm{C}}}(\theta)+\frac{1}{T} & \left((M-K) \mathbf{a}^{H}(\theta) \mathbf{U a}(\theta)\right. \\
& -\operatorname{Tr}(\mathbf{U}) g^{\left.\operatorname{Alg}_{\mathrm{C}}(\theta)\right)+o\left(\frac{1}{T}\right)}
\end{aligned}
$$

where $g^{\operatorname{Alg}_{\mathrm{C}}(\theta)} \stackrel{\text { def }}{=} \mathbf{a}^{H}(\theta) \Pi \mathbf{a}(\theta)$. For $K=2$, this expression of the mean null spectrum coincides with those given in the circular Gaussian assumption [3], [10]. Therefore, we can conclude the following result for two independent equal-power sources $\left(\sigma_{x}^{2} \stackrel{\text { def }}{=} \sigma_{x_{1}}^{2}=\sigma_{x_{2}}^{2}\right)$ where the array signal-to-noise ratio (ASNR) is defined by $r \stackrel{\text { def }}{=} M \sigma_{x}^{2} / \sigma_{n}^{2}$.

Result 1: The threshold ASNRs deduced from the Cox (3.3) and the Sharman and Durrani criterion (3.4) given for the conventional MUSIC algorithm with two independent equal-power sources do not depend on the distribution including the noncircularity of the sources.

Consequently, expressions $[3$, (rel. (35) $],{ }^{3}[9$, rel. (18)] and [6, rels. (91) and (93)] of the threshold ASNRs remain valid for arbitrary distributions of the sources. The first two expressions of this threshold ASNR dedicated to the ULA are given in the following to be compared to those derived in the next section:

$$
\begin{aligned}
& \xi_{1} \approx \frac{2}{T} \frac{\alpha_{M}}{(\Delta \theta)^{4}}\left(1+\sqrt{1+\frac{T(\Delta \theta)^{2}}{2 \beta_{M}}}\right) \\
& \xi_{2} \approx \frac{1}{T} \frac{\alpha_{M}}{(\Delta \theta)^{4}}\left(1+\sqrt{1+\frac{T(\Delta \theta)^{2}}{\beta_{M}}}\right)
\end{aligned}
$$

with $\alpha_{M} \stackrel{\text { def }}{=} 10 M^{4} /(M+2)\left(M^{2}-1\right)$ and $\beta_{M} \stackrel{\text { def }}{=} 5 M^{2} / 2(M+2)$, for which the DOA separation is defined here by $\Delta \theta \stackrel{\text { def }}{=} M\left(\theta_{1}-\theta_{2}\right) /(2 \sqrt{3})^{4}$ associated with the symmetric steering vectors $\mathbf{a}_{k}=\left(e^{-i\left((M-1) \theta_{k} / 2\right)}, e^{-i\left((M-3) \theta_{k} / 2\right)}, \ldots, e^{i\left((M-1) \theta_{k} / 2\right)}\right)^{T}$ where the coordinate system has its origin at the centroid of the array according to the parametrization of Lee et al. [6].

\section{Resolving Power of Noncircular MUSIC}

The previous approach applies to the noncircular MUSIC algorithm by replacing $\Pi, \mathbf{R}_{y, T}$ and $\mathbf{S}$ by $\tilde{\boldsymbol{\Pi}}, \mathbf{R}_{\tilde{y}, T}$ and $\tilde{\mathbf{S}}$ in (3.5), respectively. Using the following lemma proved in Appendix A.

Lemma 2: For $K$ independent rectilinear, possibly non Gaussian sources, we have

$$
\begin{aligned}
\mathrm{E}\left(\delta \mathbf{R}_{\tilde{y}, T} \mathbf{B} \delta \mathbf{R}_{\tilde{y}, T}\right)=\frac{1}{T}( & \operatorname{Tr}\left(\mathbf{B} \mathbf{R}_{\tilde{y}}\right) \mathbf{R}_{\tilde{y}}+\mathbf{R}_{\tilde{y}} \mathbf{J B}^{T} \mathbf{J R}_{\tilde{y}} \\
& \left.+\sum_{k=1}^{K} \kappa_{k} \tilde{\mathbf{a}}_{k} \tilde{\mathbf{a}}_{k}^{H} \mathbf{B} \tilde{\mathbf{a}}_{k} \tilde{\mathbf{a}}_{k}^{H}\right)+o\left(\frac{1}{T}\right)
\end{aligned}
$$

${ }^{3}$ We note that due to a mistake in the derivation of [3, (rel. (33)] and a miscalculation in a series expansion, [3, (rel. (B.2)] and [3, (rel. (35)] are erroneous. Expression (3.7) is the correct one.

${ }^{4}$ Most of the papers dealing with this topic use this normalization, so we also use it in order to simplify comparisons with the literature. 
with $\tilde{\mathbf{a}}_{k} \stackrel{\text { def }}{=}\left(\begin{array}{c}\mathbf{a}_{k} \\ \mathbf{a}_{k}^{*} e^{-2 i \phi_{k}}\end{array}\right)$, we prove in Appendix B the following expressions:

$$
\mathrm{E}\left(\delta \tilde{\mathbf{\Pi}}_{T}\right)=\frac{1}{T}(\operatorname{Tr}(\tilde{\mathbf{\Pi}}) \tilde{\mathbf{U}}-\operatorname{Tr}(\tilde{\mathbf{U}}) \tilde{\mathbf{\Pi}})+o\left(\frac{1}{T}\right)
$$

with $\tilde{\mathbf{U}} \stackrel{\text { def }}{=} \sigma_{n}^{2} \tilde{\mathbf{S}} \# \mathbf{R}_{\tilde{y}} \tilde{\mathbf{S}} \#=\left(\begin{array}{cc}\mathbf{U}_{1} & \mathbf{U}_{2} \\ \mathbf{U}_{2}^{*} & \mathbf{U}_{1}^{*}\end{array}\right)$ which gives with $\tilde{\boldsymbol{\Pi}}=\left(\begin{array}{ll}\Pi_{1} & \Pi_{2} \\ \Pi_{2}^{*} & \Pi_{1}^{*}\end{array}\right)$, Hermitian positive-semidefinite matrices

$$
\begin{aligned}
& \mathrm{E}\left(\delta \boldsymbol{\Pi}_{1, T}\right)=\frac{2}{T}\left(\operatorname{Tr}\left(\boldsymbol{\Pi}_{1}\right) \mathbf{U}_{1}-\operatorname{Tr}\left(\mathbf{U}_{1}\right) \Pi_{1}\right)+o\left(\frac{1}{T}\right) \\
& \mathrm{E}\left(\delta \boldsymbol{\Pi}_{2, T}\right)=\frac{2}{T}\left(\operatorname{Tr}\left(\boldsymbol{\Pi}_{1}\right) \mathbf{U}_{2}-\operatorname{Tr}\left(\mathbf{U}_{1}\right) \Pi_{2}\right)+o\left(\frac{1}{T}\right) .
\end{aligned}
$$

This allows us to derive the mean null spectrum associated with noncircular MUSIC algorithm (2.2). After simple but tedious algebraic manipulations summarized in Appendix C, we obtain under the assumptions of Lemma 2

$$
\begin{aligned}
& \mathrm{E}\left(g_{T}^{\operatorname{Alg}_{\mathrm{NC}}}(\theta)\right)=g^{\mathrm{Alg}_{\mathrm{NC}}(\theta)}+\frac{2}{T}(2 M-K-1) \\
& \times\left[\left(\mathbf{a}^{H}(\theta) \mathbf{U}_{1} \mathbf{a}(\theta)\right)\left(\mathbf{a}^{H}(\theta) \mathbf{\Pi}_{1} \mathbf{a}(\theta)\right)\right. \\
& \left.-\Re\left[\left(\mathbf{a}^{H}(\theta) \mathbf{U}_{2} \mathbf{a}^{*}(\theta)\right)\left(\mathbf{a}^{T}(\theta) \boldsymbol{\Pi}_{2}^{*} \mathbf{a}(\theta)\right)\right]\right]
\end{aligned}
$$

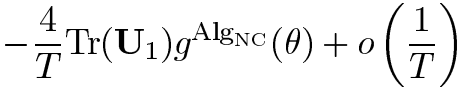

with $g^{\operatorname{Alg}_{\mathrm{NC}}}(\theta) \stackrel{\text { def }}{=}\left(\mathbf{a}^{H}(\theta) \Pi_{1} \mathbf{a}(\theta)\right)^{2}-\left|\mathbf{a}^{T}(\theta) \mathbf{\Pi}_{2}^{*} \mathbf{a}(\theta)\right|^{2}$. Since the expression of this mean null spectrum depends on the second-order statistics only, it is the same for the different threshold ASNRs deduced from it for two sources. Using closed-form expressions of $\mathbf{U}_{1}, \mathbf{U}_{2}, \boldsymbol{\Pi}_{1}$, and $\boldsymbol{\Pi}_{2}$ derived in Appendix D, the following result is proved in Appendix E after tedious algebraic manipulations.

Result 2: The threshold ASNRs deduced from the Cox (3.3) and the Sharman and Durrani (3.4) criteria given for the noncircular MUSIC algorithm with two independent equal-power sources and an arbitrary array depend only on the second-order statistics of the sources and are respectively given by

$$
\begin{aligned}
& \xi_{1} \approx \frac{2}{T} \alpha_{M}^{\Delta \theta, \Delta \phi}\left(1+\sqrt{1+\frac{T}{2 \beta_{M}^{\Delta \theta, \Delta \phi}}}\right) \\
& \xi_{2} \approx \frac{1}{T} \alpha_{M}^{\Delta \theta, \Delta \phi}\left(1+\sqrt{1+\frac{T}{\beta_{M}^{\Delta \theta, \Delta \phi}}}\right)
\end{aligned}
$$

where $\Delta \phi \stackrel{\text { def }}{=} \phi_{1}-\phi_{2}$ is the noncircularity phase separation and $\Delta \theta$ is defined according to the general parametrization of Lee et al. [6] and where $\alpha_{M}^{\Delta \theta, \Delta \phi}$ and $\beta_{M}^{\Delta \theta, \Delta \phi}$ are expansions in $1 /(\Delta \theta)^{2}$ without constant term, whose coefficients depend on $M, \Delta \phi$ and the array configuration.

We note that these threshold ASNRs (4.5) and (4.6) depend not only on $\Delta \theta, T, M$, but also on the noncircularity phase separation $\Delta \phi$, contrary to the threshold ASNRs obtained for the conventional MUSIC algorithm. Using symbolic calculus akin to a high level language (e.g., Maple software), these intricate expressions reduce to simple interpretable expressions for weak and large noncircularity phase separation $\Delta \phi$. For example, for a ULA where $\Delta \theta$ is defined as in Result 1 to simplify comparisons, we have more precisely the following approximations. First, in the case $\sin (2 \Delta \phi) \ll(M-1)(\Delta \theta / 2)$

$$
\begin{aligned}
\alpha_{M}^{\Delta \theta, \Delta \phi} & \approx \frac{5 M^{4}(2 M-3)}{\left(M^{2}-1\right)\left(M^{2}-4\right)(\Delta \theta)^{4}} \\
\beta_{M}^{\Delta \theta, \Delta \phi} & \approx \frac{5 M^{2}(2 M-3)}{2\left(M^{2}-4\right)(\Delta \theta)^{2}}
\end{aligned}
$$

and the behavior of the conventional and noncircular MUSIC algorithms are similar due to the similarity of the dependence in $\Delta \theta$ in the expressions (3.7), (3.8), (4.5), and (4.6). In the opposite case, for $\tan (\Delta \phi) \gg(M-1)(\Delta \theta / 2)$, we prove that

$$
\begin{aligned}
& \alpha_{M}^{\Delta \theta, \Delta \phi} \approx \frac{M^{2}(2 M-3)}{\left(M^{2}-1\right) \sin ^{2}(\Delta \phi)(\Delta \theta)^{2}} \\
& \beta_{M}^{\Delta \theta, \Delta \phi} \approx \frac{M^{2}(2 M-3)}{\left(M^{2}-1\right)\left(1+\cos ^{2}(\Delta \phi)\right)(\Delta \theta)^{2}}
\end{aligned}
$$

and the noncircular MUSIC algorithm largely outperforms the conventional MUSIC algorithm due to the proportionality of $\alpha_{M}^{\Delta \theta, \Delta \phi}$ in $1 /(\Delta \theta)^{2}$ in the place of $1 /(\Delta \theta)^{4}$ given in Result 1 for the conventional MUSIC algorithm. The domain of validity of these approximated expressions will be specified in the next section. Naturally, this domain depends on $\Delta \theta, M$ and the criterion, but it will be shown that this domain of validity for weak phase separations is small in contrast to those for large phase separations. For this second approximation with a large domain of validity, we note that $\alpha_{M}^{\Delta \theta, \Delta \phi}$ and $\beta_{M}^{\Delta \theta, \Delta \phi}$ are decreasing and increasing function of $\Delta \phi$ for $\Delta \phi \in] 0, \pi / 2]$, respectively. Consequently, we have proved the following result.

Result 3: The threshold ASNRs deduced from the Cox (3.3) and the Sharman and Durrani (3.4) criteria given for the noncircular MUSIC algorithm with two independent equal-power sources and a ULA are decreasing function of the phase separation of the sources for $\Delta \phi \in] 0, \pi / 2]$ and thus are minimum for $\Delta \phi=\pi / 2$.

Consequently, the noncircularity phase separation between the two sources plays an crucial role in the behavior of the noncircular MUSIC algorithm. Furthermore, we note that the expressions of $\alpha_{M}^{\Delta \theta, \Delta \phi}$ and $\beta_{M}^{\Delta \theta, \Delta \phi}$ depend of the choice of the origin of the coordinate system, in contrast to $\alpha_{M}$, and $\beta_{M}$ obtained for the conventional MUSIC algorithm. This is explained by the steering vector which has the structure $\mathbf{a}(\theta)=$ $\left(e^{i(2 \pi / \lambda) \mathbf{r}_{1}^{T} \mathbf{i}(\theta)}, e^{i(2 \pi / \lambda) \mathbf{r}_{2}^{T} \mathbf{i}(\theta)} \ldots, e^{i(2 \pi / \lambda) \mathbf{r}_{M}^{T} \mathbf{i}(\theta)}\right)^{T}$, where $\lambda$ is the wavelength and $\mathbf{r}_{m}$ and $\mathbf{i}(\theta)$ denote the vector pointing from the array centroid to sensor $m$ and the unit-length arrival vector form a source in the direction $\theta$, respectively. If the origin of the coordinate system is moved from the array centroid to the point $\mathbf{r}$, the new steering vector becomes $e^{i(2 \pi / \lambda) \mathbf{r}^{T} \mathbf{i}(\theta)} \mathbf{a}(\theta)$. Consequently, for rectilinear signals, (2.1) is rewritten as

$$
\mathbf{y}_{t}=\sum_{k=1}^{K} r_{t, k} e^{i \phi_{k}} \mathbf{a}\left(\theta_{k}\right)+\mathbf{n}_{t} \text { where } r_{t, k} \in \mathbb{R}
$$

and when the origin moves, $\mathbf{y}_{t}$ becomes

$$
\mathbf{y}_{t}=\sum_{k=1}^{K} r_{t, k} e^{i\left(\phi_{k}+\frac{2 \pi}{\lambda} \mathbf{r}^{T} \mathbf{i}\left(\theta_{k}\right)\right)} \mathbf{a}\left(\theta_{k}\right)+\mathbf{n}_{t}
$$




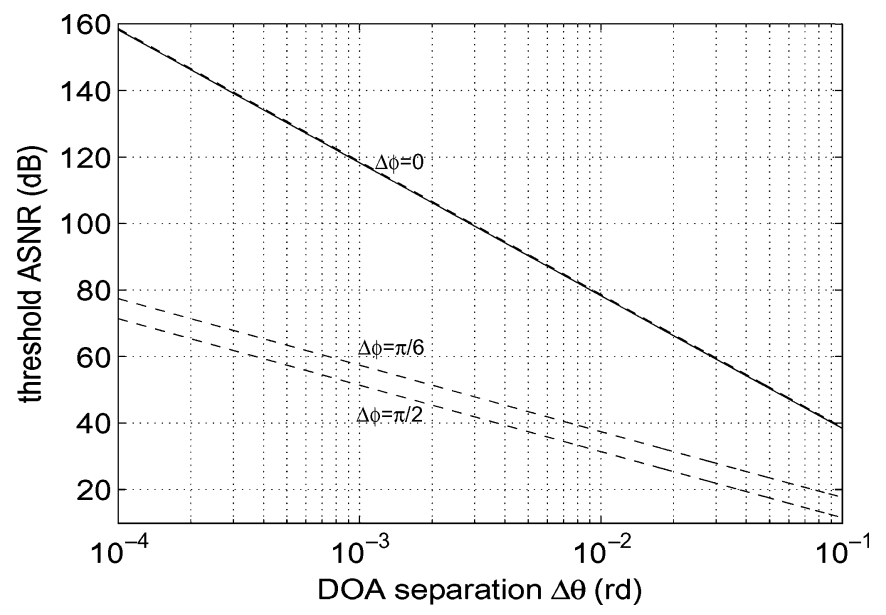

Fig. 1. Comparison of the threshold ASNRs given by the Cox criterion as a function of the DOA separation $\Delta \theta$ associated with the conventional MUSIC $(-)$ and noncircular MUSIC algorithms (- - ) for three values of the noncircularity phase separation $\Delta \phi$.

and the noncircularity phase and the DOA are coupled. This implies that this change of the origin of the coordinate system does not modify the matrices that appear in (3.6) in contrast to those of (4.1) and the resolving power of the noncircular MUSIC algorithm is sensitive to the position of this origin in contrast to the conventional MUSIC algorithm. Note that this sensitivity to the phase reference also applies to the theoretical asymptotic variance of the DOA estimated by the non-circular MUSIC algorithm. As the CRB is concerned, the noncircular Gaussian CRB [21, rel. (3.9)] in which the rectilinear property is a priori unknown does not depend on the phase reference of the array in contrast to the noncircular Gaussian CRB that takes this rectilinear property as a priori known.

This quite curious result reminds one of a similar result in cissoid parameter estimation (see, e.g., [22] and [23, pp. 273-286]) where the Cramér-Rao bounds of the frequency and phase depend upon the time at which the first sample is taken and are minimum if the sampling times are symmetrically located about zero. So, the centroid of the array that has been chosen as phase origin to simplify the notation, can be conjectured to be the phase origin that optimizes the performance of estimation and resolution of closely spaced rectilinear sources.

\section{ILLUSTRATIVE EXAMPLES}

To illustrate Results 1, 2, and 3, we consider throughout this section two independent equal-power BPSK modulated signals impinging on a ULA of sensors with $M=10$ and $T=500$ (except for Figs. 9 and 10). We clearly see in Figs. 1 and 2 that the noncircular MUSIC algorithm outperforms the conventional MUSIC algorithm except for very weak noncircularity phase separations for which the ASNR thresholds of these two algorithms are very similar. Furthermore, we note that the behaviors of the ASNR threshold given by the two criteria are very similar although the ASNR thresholds are slightly weaker for the Sharman and Durrani criterion than for the Cox criterion. This is explained by the bias of the sample null spectrum at the true source direction that could imply that although the

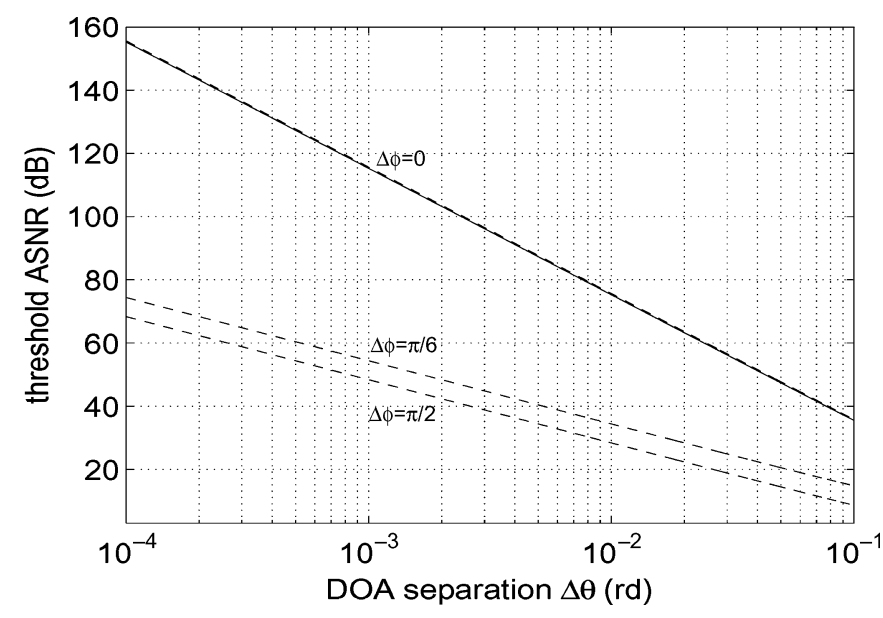

Fig. 2. Comparison of the ASNR thresholds given by the Sharman and Durrani criterion as a function of the DOA separation $\Delta \theta$ associated with the conventional MUSIC ( - ) and noncircular MUSIC algorithms $(--)$ for three values of the noncircularity phase separation $\Delta \phi$.

midpoint mean null spectrum is less than the mean of the mean null spectrum in the true directions, the algorithm recognizes two sources because the Sharman and Durrani criterion is satisfied (see, e.g., [8, Fig. 3]). Finally, we cheek from these two figures that the threshold ASNRs are approximately to $1 /(\Delta \theta)^{4}$ for the noncircular MUSIC algorithm with $\Delta \phi=\pi / 6$ and $\Delta \phi=\pi / 2$, in contrast to the conventional and noncircular MUSIC algorithm with $\Delta \phi=0$, for which they are approximately to $1 /(\Delta \theta)^{2}$.

Figs. 3 and 4 exhibit the domain of validity of the approximations of the threshold ASNRs given in the foregoing section for weak and large noncircularity phase separations $\Delta \phi$. From these figures given for $M=10$, these approximations are valid in a large domain of $\Delta \phi$ for the second approximation (for which this domain enlarges when $\Delta \theta$ decreases which is consistent with the condition $\tan (\Delta \phi) \gg(M-1)(\Delta \theta / 2))$ in contrast to the first approximation (for which it enlarges when $\Delta \theta$ increases which is consistent with $\sin (2 \Delta \phi) \ll(M-1)(\Delta \theta / 2))$. We note that the domains of validity of these approximations are larger for the Sharman and Durrani criterion than for the Cox criterion.

Figs. 5 and 6 show the probability of resolution related to the foregoing two criteria obtained by Monte Carlo simulations. ${ }^{5}$ Compared with the ASNR thresholds given by Figs. 1 and 2, we see that the ASNR threshold given by our non probabilistic approach based on the mean null spectrum $g_{T}(\theta)$ corresponds to a probability of resolution that ranges from 0.3 to 0.7 for the two criteria. Thus, this non probabilistic approach gives correct approximate ASNR for the $0.3-0.7$ probability of resolution threshold region in the same way that for the conventional MUSIC algorithm [3]. Furthermore, we note that the resolution is much more sensitive to the ASNR for the Sharman and Durrani criterion than for the Cox criterion.

\footnotetext{
${ }^{5}$ In each simulation trial, the two sources are considered resolved for these two criteria if $\sup \left(g_{T_{\mathrm{NC}}}^{\text {Alg }}\left(\theta_{1}\right), g_{T}^{\mathrm{Alg} N \mathrm{NC}}\left(\theta_{2}\right)\right)<g_{T}^{\mathrm{Alg} N \mathrm{C}}\left(\theta_{m}\right)$ for the Cox criterion, and $g_{T}^{\operatorname{Alg}_{N C}}\left(\theta_{m}+0.002 \Delta \theta\right)+g_{T}^{\operatorname{Alg}_{N C}}\left(\theta_{m}-\right.$ $0.002 \Delta \theta)-2 g_{T}^{\mathrm{Alg}_{\mathrm{NC}}}\left(\theta_{m}\right)<0$ for the Sharman and Durrani criterion.
} 


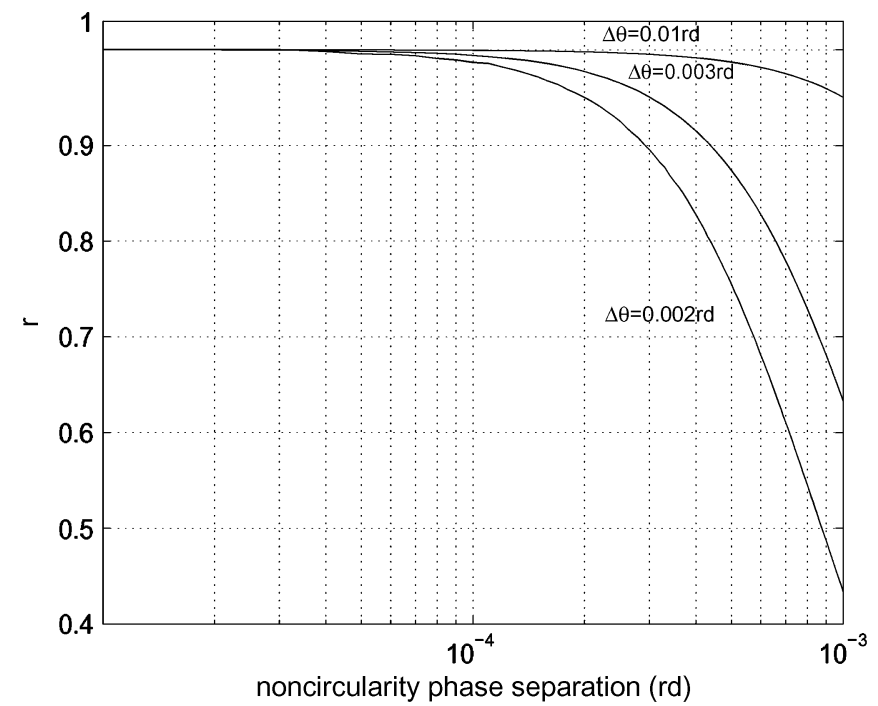

(a)

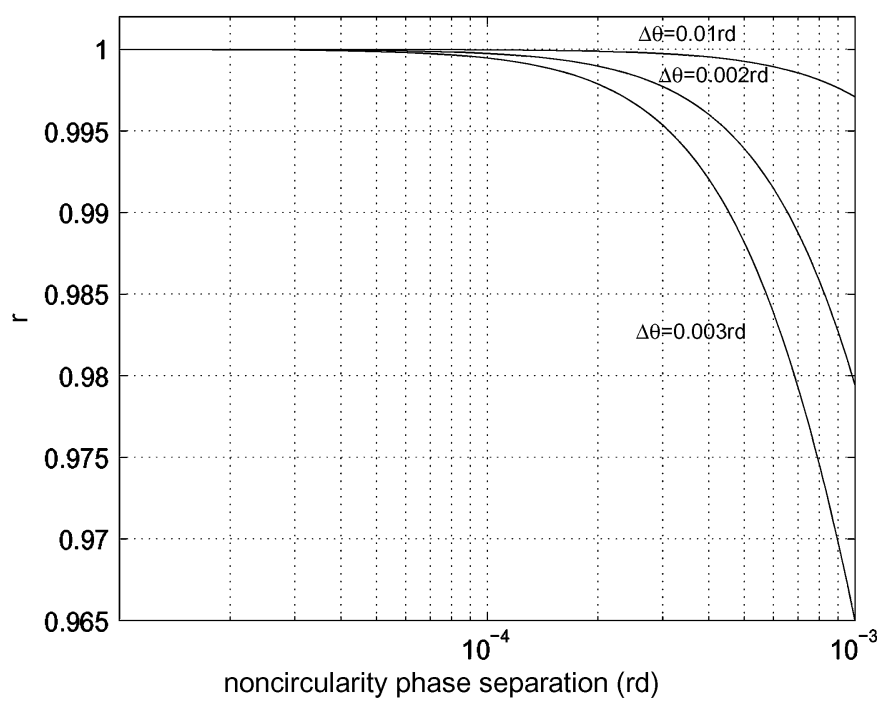

(b)

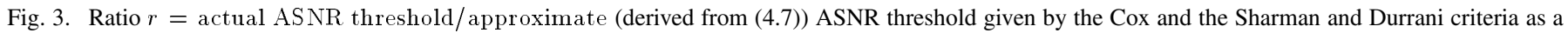
function of the noncircularity phase separation $\Delta \phi$ for three DOA separations $\Delta \theta$. (a) Cox criterion and (b) Sharman and Durrani criterion.

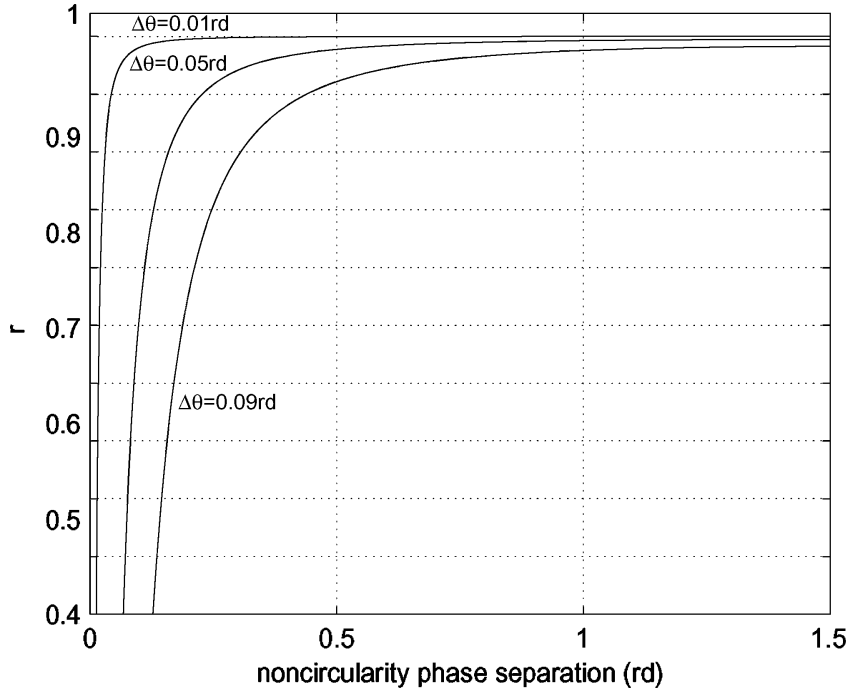

(a)

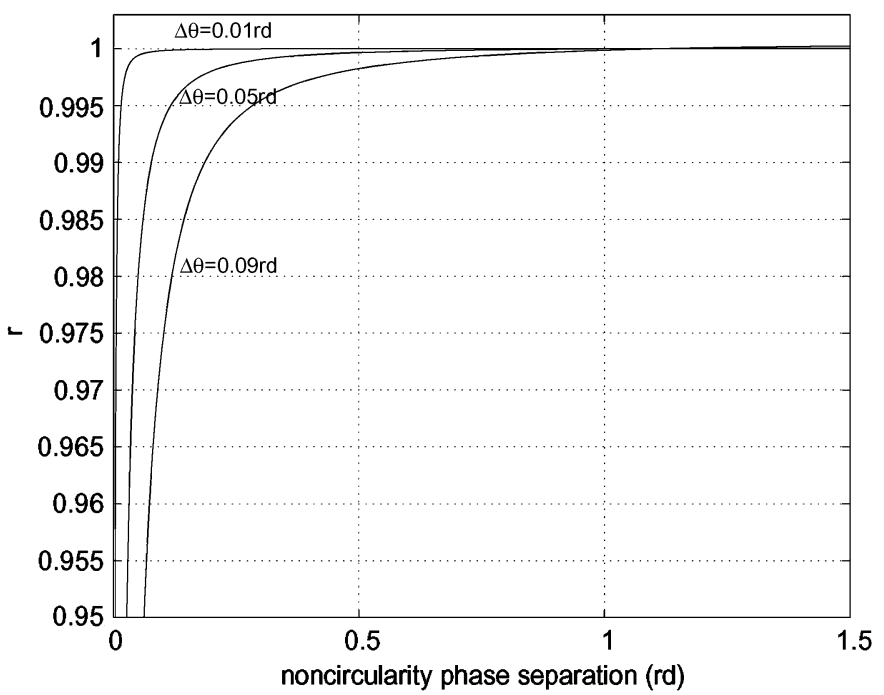

(b)

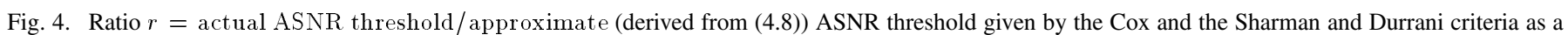
function of the noncircularity phase separation $\Delta \phi$ for three DOA separations $\Delta \theta$. (a) Cox criterion and (b) Sharman and Durrani criterion.

We note that the difference of behavior in resolution between the conventional and noncircular MUSIC algorithms are connected to the accuracy of the DOA estimate given by the noncircular MUSIC algorithm [17] compared to those of the conventional MUSIC algorithm. This is illustrated in Figs. 7 and 8 where the variance of the estimated DOA and of the separation of DOA are respectively exhibited. In these figures, the theoretical asymptotic variances are derived form the covariance of the asymptotic distribution of the DOAs estimated by the conventional and the noncircular MUSIC given in [17]. We note in particular that the performances given by the noncircular MUSIC algorithms for $\Delta \phi=0$ and those of the conventional MUSIC algorithms are theoretically and empirically different but very closed.
Finally, we question the domain of validity of our approach that is totally based on the contention that the mean value of the sample null spectrum can be taken as representative of the ensemble of sample null spectra obtained for a large number $T$ of snapshots and a fixed number $M$ of sensors and more specifically under the condition $T \gg M \gg 1$. In Figs. 9 and 10, $M=10$ and $\Delta \theta=0.1 \mathrm{rd}$ are fixed, in contrast to the number $T$ of snapshots. First, we cheek that because $T \ll 2 \beta_{M}^{\Delta \theta, \Delta \phi}$, the ASNR threshold varies as $(2 / T) \alpha_{M}^{\Delta \theta, \Delta \phi}$. Then compared with the ASNR threshold given by Fig. 9, we see in Fig. 10 that the ASNR threshold given by our approach based on the mean null spectrum $g_{T}(\theta)$ corresponds to a probability of resolution that keeps on ranging from 0.2 to 0.7 for the Cox criterion provided that $T \leq 7$. Consequently our approach seems to be valid at least in the domain $M \gg 1$ and $T / M \geq 1$. Consequently, the 


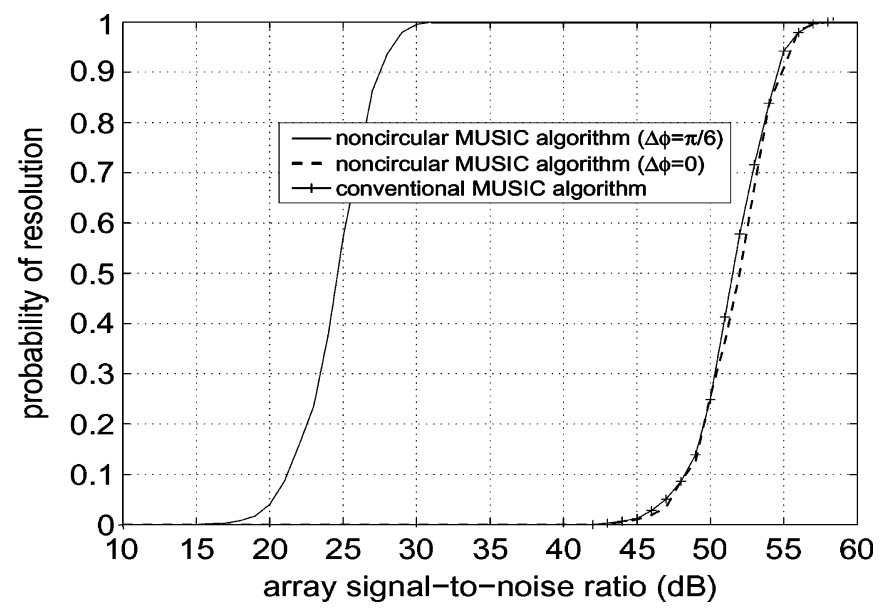

Fig. 5. Probability of resolution given by the Cox criterion (Monte Carlo with 1000 runs) as function of the ASNR for $\Delta \theta=0.05 \mathrm{rd}$ for the noncircular MUSIC algorithm compared to the conventional MUSIC algorithm.

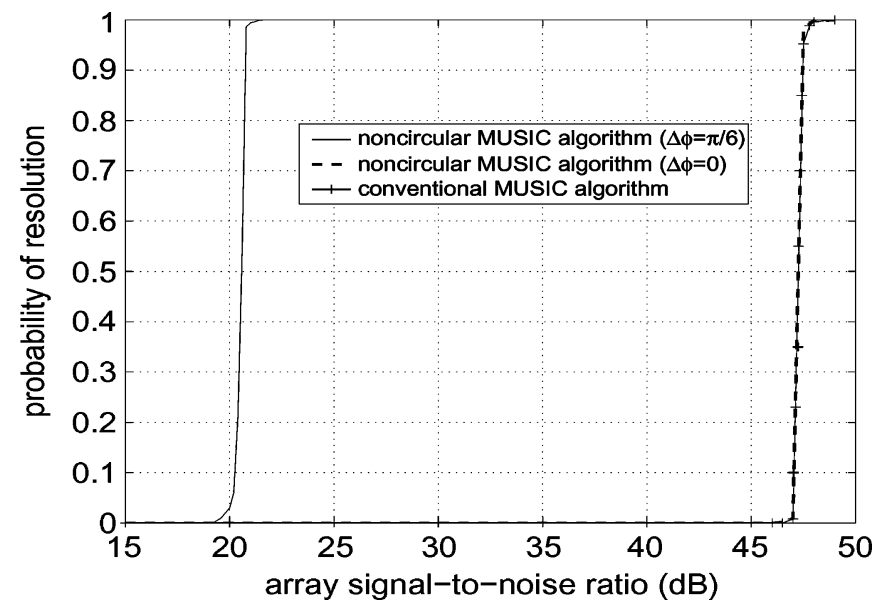

Fig. 6. Probability of resolution given by the Sharman and Durrani criterion (Monte Carlo with 1000 runs) as function of the ASNR for $\Delta \theta=0.05 \mathrm{rd}$ for the noncircular MUSIC algorithm compared to the conventional MUSIC algorithm.

Cox and the Sharman and Durrani criteria that are very close, enable one to obtain a coarse estimate of the ASNR threshold in a relatively large domain of validity.

\section{CONCLUSION}

This paper has presented a theoretical analysis of the resolution according to the Cox and the Sharman and Durrani criteria for the conventional and noncircular MUSIC algorithms for arbitrary circular and noncircular second-order distribution of two closely spaced transmitters. It has been proved that these threshold ASNRs given by the conventional MUSIC algorithm do not depend on the distribution of the sources including their noncircularity, in contrast to the noncircular MUSIC algorithm for which they are very sensitive to the noncircularity phase separation of the sources. More precisely, it has been proved that for very weak phase separations the behavior of the conventional and noncircular MUSIC algorithms are very similar, in contrast to large phase separation for which the noncircular

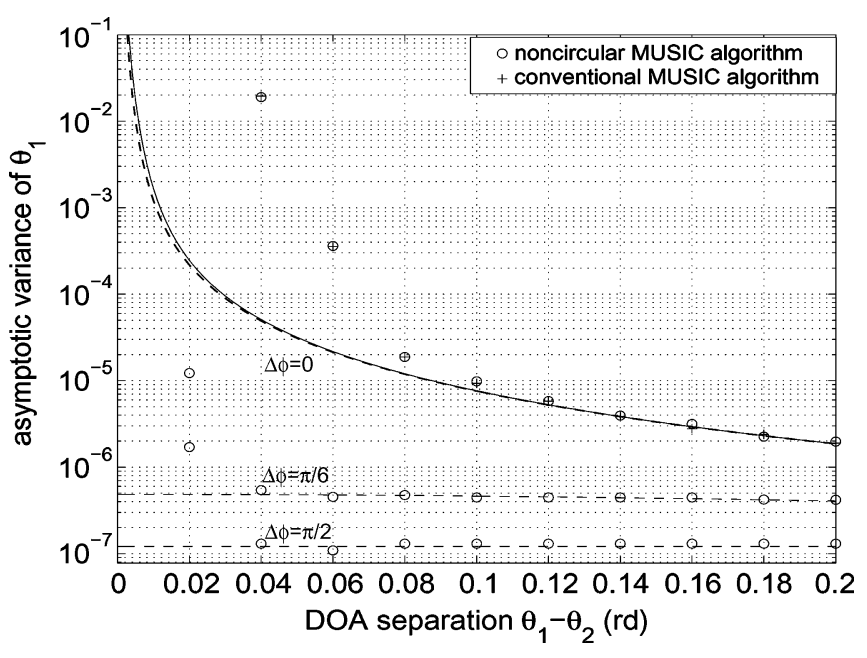

Fig. 7. Theoretical asymptotic variance of the DOA estimates given by the conventional MUSIC ( -$)$ and the noncircular MUSIC $(--)$ algorithms and empirical variance given by the conventional MUSIC $(+)$ and the noncircular MUSIC (o) algorithms (with 1000 Monte Carlo runs) for three values of the noncircularity phase separation $\Delta \phi$ as a function of the DOA separation $\Delta \theta$, for $\mathrm{SNR}=20 \mathrm{~dB}$.

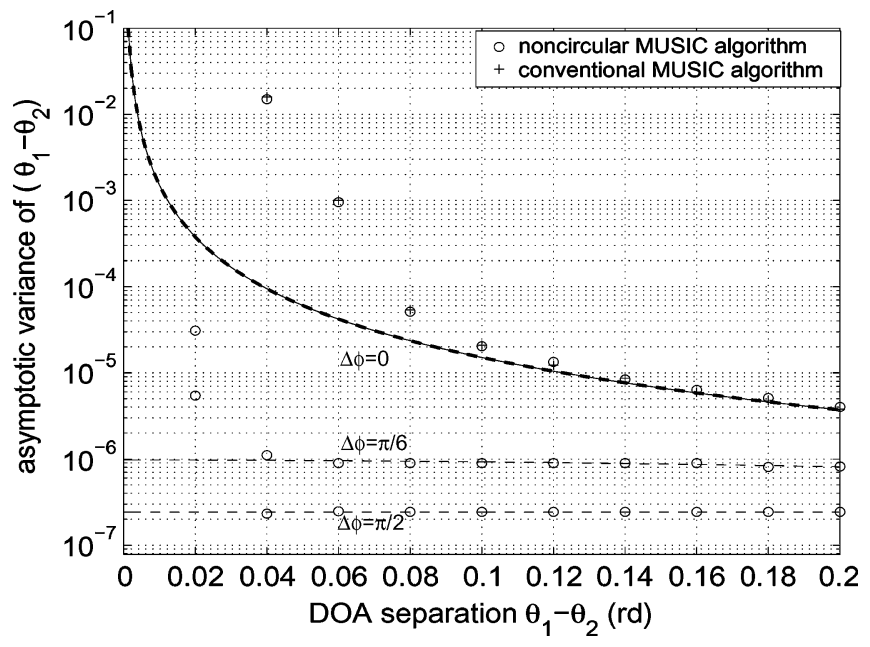

Fig. 8. Theoretical asymptotic variance of the difference between DOA estimates given by the conventional MUSIC (-) and the noncircular MUSIC $(--)$ algorithms and empirical variance of the difference between DOA estimates given by the conventional MUSIC $(+)$ and the noncircular MUSIC (o) algorithms (with 1000 Monte Carlo runs) for three values of the noncircularity phase separation $\Delta \phi$ as a function of the DOA separation $\Delta \theta$, for $\mathrm{SNR}=20 \mathrm{~dB}$.

MUSIC algorithm comfortably outperforms the conventional MUSIC algorithm.

\section{APPENDIX A}

PROOF OF LEMMAS 1 AND 2

We recall for the ease of the reader the following identities that will be frequently used in all Appendices (see, e.g., [24, Th. 7.7, 7.16, and 7.17]):

$$
\begin{aligned}
(\mathbf{A} \otimes \mathbf{B})(\mathbf{C} \otimes \mathbf{D}) & =\mathbf{A} \mathbf{C} \otimes \mathbf{B D} \\
\operatorname{vec}(\mathbf{A B C}) & =\left(\mathbf{C}^{T} \otimes \mathbf{A}\right) \operatorname{vec}(\mathbf{B}) \\
\operatorname{Tr}(\mathbf{A B C D}) & =\operatorname{vec}^{T}\left(\mathbf{A}^{T}\right)\left(\mathbf{D}^{T} \otimes \mathbf{B}\right) \operatorname{vec}(\mathbf{C}) .
\end{aligned}
$$




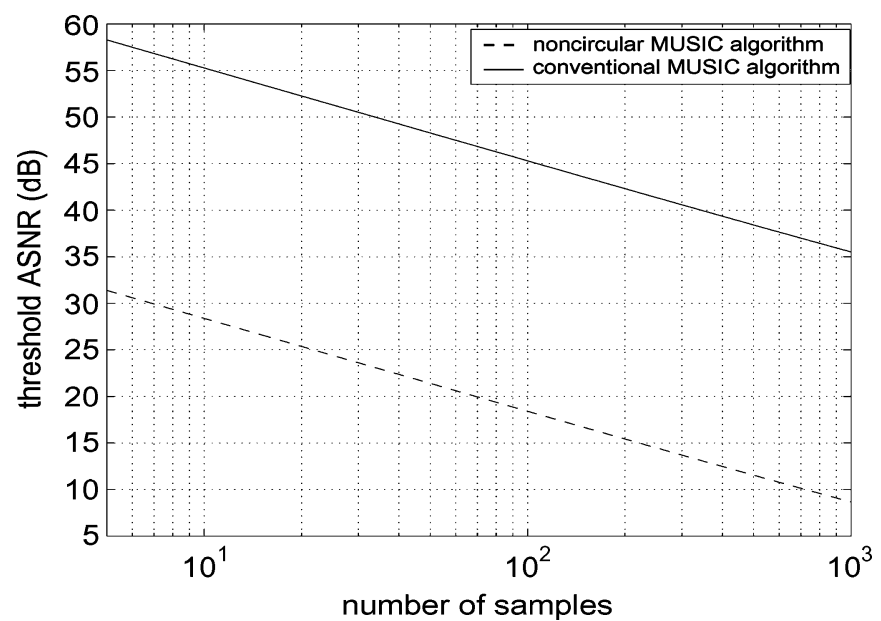

Fig. 9. Comparison of the threshold ASNRs given by the Cox criterion as a function of the number $T$ of snapshots associated with the conventional MUSIC $(-)$ and noncircular MUSIC algorithms $(--)$ for $\Delta \theta=0.1 \mathrm{rd}$ and $\Delta \phi=$ $\pi / 2 \mathrm{rd}$.

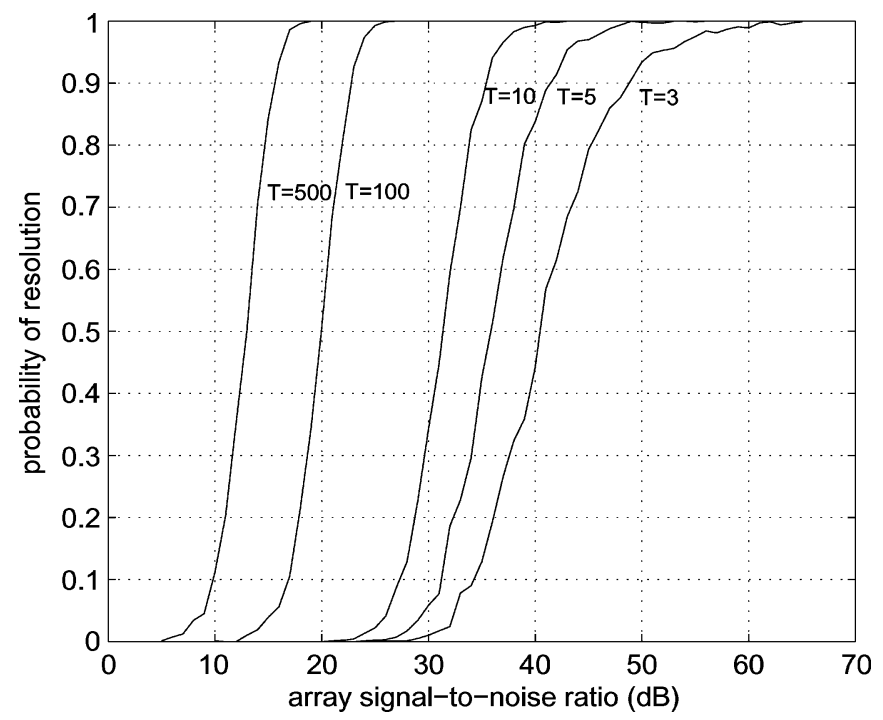

Fig. 10. Probability of resolution given by the Cox criterion (Monte Carlo with 1000 runs) as function of the ASNR for $\Delta \theta=0.1 \mathrm{rd}$ for the noncircular MUSIC algorithm with $\Delta \phi=\pi / 2 \mathrm{rd}$, for six values of the number $T$ of snapshots.

First, using the vectorization operator and (A.2), we have

$$
\operatorname{vec}\left(\mathrm{E}\left(\delta \mathbf{R}_{y, T} \mathbf{B} \delta \mathbf{R}_{y, T}\right)\right)=\mathrm{E}\left(\delta \mathbf{R}_{y, T}^{T} \otimes \delta \mathbf{R}_{y, T}\right) \operatorname{vec}(\mathbf{B})
$$

with

$$
\begin{aligned}
\mathrm{E} & \left(\delta \mathbf{R}_{y, T}^{T} \otimes \delta \mathbf{R}_{y, T}\right) \\
& =\frac{1}{T^{2}} \mathrm{E}\left(\sum_{t=1}^{T} \sum_{t^{\prime}=1}^{T}\left(\mathbf{y}_{t}^{*} \mathbf{y}_{t}^{T}-\mathbf{R}_{y}^{T}\right) \otimes\left(\mathbf{y}_{t^{\prime}} \mathbf{y}_{t^{\prime}}^{H}-\mathbf{R}_{y}\right)\right) \\
& =\frac{1}{T} \mathrm{E}\left(\left(\mathbf{y}_{t}^{*} \mathbf{y}_{t}^{T}-\mathbf{R}_{y}^{T}\right) \otimes\left(\mathbf{y}_{t} \mathbf{y}_{t}^{H}-\mathbf{R}_{y}\right)\right) \\
& =\frac{1}{T}\left(\mathrm{E}\left(\mathbf{y}_{t}^{*} \mathbf{y}_{t}^{T} \otimes \mathbf{y}_{t} \mathbf{y}_{t}^{H}\right)-\mathbf{R}_{y}^{T} \otimes \mathbf{R}_{y}\right)
\end{aligned}
$$

where $\mathbf{y}_{t}^{*} \mathbf{y}_{t}^{T} \otimes \mathbf{y}_{t} \mathbf{y}_{t}^{H}=\left(\mathbf{y}_{t}^{*} \otimes \mathbf{y}_{t}\right)\left(\mathbf{y}_{t}^{T} \otimes \mathbf{y}_{t}^{H}\right)=$ $\operatorname{vec}\left(\mathbf{y}_{t} \mathbf{y}_{t}^{H}\right) \operatorname{vec}^{H}\left(\mathbf{y}_{t} \mathbf{y}_{t}^{H}\right)$. Using the following relation deduced from the derivation of [25] in the particular case of independent sources:

$$
\begin{gathered}
\mathrm{E}\left[\operatorname{vec}\left(\mathbf{y}_{t} \mathbf{y}_{t}^{H}\right) \operatorname{vec}^{H}\left(\mathbf{y}_{t} \mathbf{y}_{t}^{H}\right)\right]-\operatorname{vec}\left(\mathbf{R}_{y}\right) \operatorname{vec}^{H}\left(\mathbf{R}_{y}\right) \\
=\mathbf{R}_{y}^{T} \otimes \mathbf{R}_{y}+\mathbf{K}\left(\mathbf{R}_{y}^{\prime} \otimes \mathbf{R}_{y}^{* *}\right)+\sum_{k=1}^{K} \kappa_{k}\left(\mathbf{A}^{*} \otimes \mathbf{A}\right) \\
\times\left(\mathbf{e}_{K, k} \otimes \mathbf{e}_{K, k}\right)\left(\mathbf{e}_{K, k}^{T} \otimes \mathbf{e}_{K, k}^{T}\right)\left(\mathbf{A}^{T} \otimes \mathbf{A}^{H}\right)
\end{gathered}
$$

we obtain

$$
\begin{aligned}
& \mathrm{E}\left(\delta \mathbf{R}_{y, T}^{T} \otimes \delta \mathbf{R}_{y, T}\right) \\
&=\frac{1}{T}\left(\operatorname{vec}\left(\mathbf{R}_{y}\right) \operatorname{vec}^{H}\left(\mathbf{R}_{y}\right)+\mathbf{K}\left(\mathbf{R}_{y}^{\prime} \otimes \mathbf{R}_{y}^{\prime *}\right)\right. \\
& \quad+\sum_{k=1}^{K} \kappa_{k}\left(\mathbf{A}^{*} \otimes \mathbf{A}\right)\left(\mathbf{e}_{K, k} \otimes \mathbf{e}_{K, k}\right) \\
&\left.\left.\quad \times\left(\mathbf{e}_{K, k}^{T} \otimes \mathbf{e}_{K, k}^{T}\right)\right)\left(\mathbf{A}^{T} \otimes \mathbf{A}^{H}\right)\right) .
\end{aligned}
$$

Incorporating this relation in (A.4) gives

$$
\begin{aligned}
& \operatorname{vec}(\left.\mathrm{E}\left(\delta \mathbf{R}_{y, T} \mathbf{B} \delta \mathbf{R}_{y, T}\right)\right) \\
&=\frac{1}{T}\left(\operatorname{vec}\left(\mathbf{R}_{y}\right)\left[\operatorname{vec}^{H}\left(\mathbf{R}_{y}\right) \operatorname{vec}(\mathbf{B})\right]\right. \\
&+ \mathbf{K}\left(\mathbf{R}_{y}^{\prime} \otimes \mathbf{R}_{y}^{\prime *}\right) \operatorname{vec}(\mathbf{B}) \\
&\left.+\sum_{k=1}^{K} \kappa_{k}\left(\mathbf{A}^{*} \otimes \mathbf{A}\right)\left(\mathbf{e}_{K, k} \otimes \mathbf{e}_{K, k}\right)\left(\mathbf{e}_{K, k}^{T} \otimes \mathbf{e}_{K, k}^{T}\right)\right) \\
&\left.\times\left(\mathbf{A}^{T} \otimes \mathbf{A}^{H}\right) \operatorname{vec}(\mathbf{B})\right)
\end{aligned}
$$

and using (A.1), (A.2), and (A.3) and $\mathbf{A e}_{K, k}=\mathbf{a}_{k}$ with $\mathbf{A} \stackrel{\text { def }}{=}$ $\left[\mathbf{a}_{1}, \ldots, \mathbf{a}_{k}\right]$ proves Lemma 1 .

For $K$ independent rectilinear sources, $x_{k, t}^{*}=e^{-2 i \phi_{k}} x_{k, t}$, $k=1, \ldots, K$ implies that $\tilde{\mathbf{y}}_{t}=\sum_{k=1}^{K} x_{k, t} \tilde{\mathbf{a}}_{k}+\tilde{\mathbf{n}}_{t}$ and consequently the proof of Lemma 1 extends by replacing $\mathbf{R}_{y, T}$, $\mathbf{R}_{y}, \mathbf{R}_{y}^{\prime}$ and $\mathbf{a}_{k}$ by $\mathbf{R}_{\tilde{y}, T}, \mathbf{R}_{\tilde{y}}, \mathbf{R}_{\tilde{y}}^{\prime}$ and $\tilde{\mathbf{a}}_{k}$, respectively. Since $\mathbf{R}_{\tilde{y}}^{\prime} \stackrel{\text { def }}{=} \mathrm{E}\left(\tilde{\mathbf{y}} \tilde{\mathbf{y}}^{T}\right)=\mathbf{R}_{\tilde{y}} \mathbf{J}$, Lemma 2 is proved.

\section{APPENDIX B}

PROOF OF RELATIONS (3.6) AND (4.1)

First, note that the first order term of (3.5) in $\mathrm{E}\left(\delta \Pi_{T}\right)$ vanishes because $\mathrm{E}\left(\delta \mathbf{R}_{y, T}\right)=\mathbf{O}$. Then, using Lemma 1 with $\mathbf{B}=\boldsymbol{\Pi}$, $\mathbf{S}^{\#}$ and $\mathbf{S}^{\#}$, the contributions of $\operatorname{Tr}\left(\mathbf{B R} \mathbf{R}_{y}\right) \mathbf{R}_{y}$ in $\mathrm{E}\left(\delta \boldsymbol{\Pi}_{T}\right)$ vanishes except the following first two terms because $\mathbf{R}_{y} \boldsymbol{\Pi}=\sigma_{n}^{2} \boldsymbol{\Pi}$ and $\mathbf{S}^{\#} \mathbf{I}=\mathbf{O}$ :

$$
\begin{aligned}
\mathbf{S}^{\#} \operatorname{Tr}\left(\boldsymbol{\Pi} \mathbf{R}_{y}\right) \mathbf{R}_{y} \mathbf{S}^{\#} & =\operatorname{Tr}\left(\sigma_{n}^{2} \boldsymbol{\Pi}\right) \mathbf{S}^{\#} \mathbf{R}_{y} \mathbf{S}^{\#}=\operatorname{Tr}(\boldsymbol{\Pi}) \mathbf{U} \\
-\boldsymbol{\Pi} \operatorname{Tr}\left(\mathbf{S}^{\# 2} \mathbf{R}_{y}\right) \mathbf{R}_{y} \boldsymbol{\Pi} & =-\boldsymbol{\Pi} \operatorname{Tr}\left(\mathbf{S}^{\#} \mathbf{R}_{y} \mathbf{S}^{\#}\right) \mathbf{R}_{y} \boldsymbol{\Pi}=-\operatorname{Tr}(\mathbf{U}) \boldsymbol{\Pi} .
\end{aligned}
$$

Finally after a close examination, all the contributions of $\mathbf{R}_{y}^{\prime} \mathbf{B}^{T} \mathbf{R}_{y}^{\prime *}$ and $\mathbf{a}_{k} \mathbf{a}_{k}^{H} \mathbf{B} \mathbf{a}_{k} \mathbf{a}_{k}^{H}$ in $\mathrm{E}\left(\delta \Pi_{T}\right)$ vanishes because $\Pi \mathbf{R}_{y}^{\prime}=\mathbf{O}$ and $\Pi \mathbf{a}_{k}=\mathbf{0}$ respectively, and consequently (3.6) is proved.

Relation (4.1) is proved in the same way by replacing $\mathbf{R}_{y}$, $\Pi$ and $\mathbf{S}$ by $\mathbf{R}_{\tilde{y}}, \tilde{\boldsymbol{\Pi}}$ and $\tilde{\mathbf{S}}$, respectively, and using the relations 
$\mathbf{J} \tilde{\Pi}^{T} \mathbf{J}=\tilde{\mathbf{\Pi}}, \mathbf{J} \tilde{\mathbf{S}}^{\#^{T}} \mathbf{J}=\tilde{\mathbf{S}}^{\#}$ and $\mathbf{J} \tilde{\mathbf{S}}^{\# 2^{T}} \mathbf{J}=\tilde{\mathbf{S}}^{\# 2}$ because $\tilde{\mathbf{S}}^{\#}$ and $\tilde{\mathbf{S}}^{\# 2}$ are structured as $\tilde{\mathbf{\Pi}}$ in the form $\left(\begin{array}{cc}(\square) & (\times) \\ (\times)^{*} & (\square)^{*}\end{array}\right)$.

APPENDIX C

PROOF OF RELATION (4.4)

To simplify the expression of $\mathrm{E}\left(g_{T}^{\mathrm{Alg}_{\mathrm{NC}}}(\theta)\right)$, introduce $\mathbf{M} \stackrel{\text { def }}{=}$ $\mathbf{a}(\theta) \mathbf{a}^{H}(\theta)$ and $\mathbf{N} \stackrel{\text { def }}{=} \mathbf{a}(\theta) \mathbf{a}^{T}(\theta)$ for which we have

$g_{T}^{\operatorname{Alg}_{\mathrm{NC}}}(\theta)=\operatorname{Tr}\left(\mathbf{\Pi}_{1, T} \mathbf{M}\right) \operatorname{Tr}\left(\mathbf{\Pi}_{1, T} \mathbf{M}\right)$

$$
-\operatorname{Tr}\left(\mathbf{\Pi}_{2, T}^{*} \mathbf{N}\right) \operatorname{Tr}\left(\mathbf{\Pi}_{2, T} \mathbf{N}^{*}\right)
$$

which gives

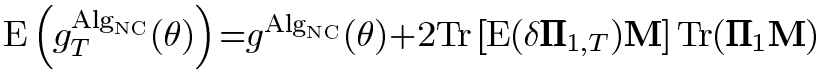

$$
\begin{aligned}
& -2 \Re\left(\operatorname{Tr}\left[\mathrm{E}\left(\delta \boldsymbol{\Pi}_{2, T}\right) \mathbf{N}^{*}\right] \operatorname{Tr}\left(\boldsymbol{\Pi}_{2}^{*} \mathbf{N}\right)\right) \\
& +\mathrm{E}\left[\operatorname{Tr}\left(\delta \boldsymbol{\Pi}_{1, T} \mathbf{M}\right) \operatorname{Tr}\left(\delta \boldsymbol{\Pi}_{1, T} \mathbf{M}\right)\right] \\
& -\mathrm{E}\left[\operatorname{Tr}\left(\delta \boldsymbol{\Pi}_{2, T}^{*} \mathbf{N}\right) \operatorname{Tr}\left(\delta \boldsymbol{\Pi}_{2, T} \mathbf{N}^{*}\right)\right]+o\left(\frac{1}{T}\right) \text {. }
\end{aligned}
$$

The first-order terms of (C.1) are deduced from (4.2) and (4.3) which gives from $\operatorname{Tr}[\tilde{\boldsymbol{\Pi}})=2 \operatorname{Tr}\left[\boldsymbol{\Pi}_{1}\right)=2 M-K$ where $\mathbf{a} \stackrel{\text { def }}{=}$ $\mathbf{a}(\theta)$ for the sake of brevity.

$$
\begin{aligned}
\operatorname{Tr}\left[\mathrm{E}\left(\delta \mathbf{\Pi}_{1, T}\right) \mathbf{M}\right]=\frac{1}{T}( & (2 M-K)\left(\mathbf{a}^{H} \mathbf{U}_{1} \mathbf{a}\right) \\
& \left.-2 \operatorname{Tr}\left(\mathbf{U}_{1}\right)\left(\mathbf{a}^{H} \mathbf{\Pi}_{1} \mathbf{a}\right)\right)+o\left(\frac{1}{T}\right) \\
\operatorname{Tr}\left[\mathrm{E}\left(\delta \boldsymbol{\Pi}_{2, T}\right) \mathbf{N}^{*}\right]=\frac{1}{T}( & (2 M-K)\left(\mathbf{a}^{H} \mathbf{U}_{2} \mathbf{a}^{*}\right) \\
& \left.-2 \operatorname{Tr}\left(\mathbf{U}_{1}\right) \mathbf{a}^{H} \mathbf{\Pi}_{2} \mathbf{a}^{*}\right)+o\left(\frac{1}{T}\right) .
\end{aligned}
$$

Introducing these expressions, the first-order terms of (C.1) are given by

$$
\begin{gathered}
2 \operatorname{Tr}\left[\mathrm{E}\left(\delta \boldsymbol{\Pi}_{1, T}\right) \mathbf{M}\right] \operatorname{Tr}\left(\boldsymbol{\Pi}_{1} \mathbf{M}\right) \\
=\frac{2}{T}\left((2 M-K)\left(\mathbf{a}^{H} \mathbf{U}_{1} \mathbf{a}\right)\left(\mathbf{a}^{H} \mathbf{\Pi}_{1} \mathbf{a}\right)\right. \\
\left.\quad-2 \operatorname{Tr}\left(\mathbf{U}_{1}\right)\left(\mathbf{a}^{H} \boldsymbol{\Pi}_{1} \mathbf{a}\right)^{2}\right)+o\left(\frac{1}{T}\right) \\
2 \Re\left(\operatorname{Tr}\left[\mathrm{E}\left(\delta \boldsymbol{\Pi}_{2, T}\right) \mathbf{N}^{*}\right] \operatorname{Tr}\left(\boldsymbol{\Pi}_{2}^{*} \mathbf{N}\right)\right) \\
=\frac{2}{T}\left((2 M-K) \Re\left(\left(\mathbf{a}^{H} \mathbf{U}_{2} \mathbf{a}^{*}\right)\left(\mathbf{a}^{T} \Pi_{2}^{*} \mathbf{a}\right)\right)\right. \\
\left.\quad-2 \operatorname{Tr}\left(\mathbf{U}_{1}\right)\left|\mathbf{a}^{H} \Pi_{2} \mathbf{a}^{*}\right|^{2}\right)+o\left(\frac{1}{T}\right)
\end{gathered}
$$

The second-order terms of (C.1) are given by

$$
\begin{aligned}
\mathrm{E} & {\left[\operatorname{Tr}\left(\delta \boldsymbol{\Pi}_{1, T} \mathbf{M}\right) \operatorname{Tr}\left(\delta \boldsymbol{\Pi}_{1, T} \mathbf{M}\right)\right] } \\
& =\operatorname{vec}^{T}\left(\mathbf{M}^{T}\right) \mathrm{E}\left(\operatorname{vec}\left(\delta \boldsymbol{\Pi}_{1, T}\right) \operatorname{vec}^{H}\left(\delta \boldsymbol{\Pi}_{1, T}\right)\right) \operatorname{vec}(\mathbf{M}) \\
& =\frac{1}{T} \operatorname{vec}^{T}\left(\mathbf{M}^{T}\right) \mathbf{C}_{\Pi_{1}} \operatorname{vec}(\mathbf{M})+o\left(\frac{1}{T}\right)
\end{aligned}
$$

$$
\begin{aligned}
\mathrm{E} & {\left[\operatorname{Tr}\left(\delta \boldsymbol{\Pi}_{2, T}^{*} \mathbf{N}\right) \operatorname{Tr}\left(\delta \boldsymbol{\Pi}_{2, T} \mathbf{N}^{*}\right)\right] } \\
& =\operatorname{vec}^{T}(\mathbf{N}) \mathrm{E}\left(\operatorname{vec}\left(\delta \boldsymbol{\Pi}_{2, T}^{*}\right) \operatorname{vec}^{T}\left(\delta \boldsymbol{\Pi}_{2, T}\right)\right) \operatorname{vec}\left(\mathbf{N}^{*}\right) \\
& =\frac{1}{T} \operatorname{vec}^{T}(\mathbf{N}) \mathbf{C}_{\Pi_{2}}^{*} \operatorname{vec}\left(\mathbf{N}^{*}\right)+o\left(\frac{1}{T}\right),
\end{aligned}
$$

where $\mathbf{C}_{\Pi_{1}}$ and $\mathbf{C}_{\Pi_{2}}$ denote the covariance matrices of the asymptotic distribution of $\Pi_{1, T}$ and $\Pi_{2, T}$, respectively, whose following expressions are proved in [17]:

$$
\begin{aligned}
\mathbf{C}_{\Pi_{1}}= & \left(\boldsymbol{\Pi}_{1}^{*} \otimes \mathbf{U}_{1}\right)+\left(\mathbf{U}_{1}^{*} \otimes \boldsymbol{\Pi}_{1}\right) \\
& +\mathbf{K}\left[\left(\boldsymbol{\Pi}_{2} \otimes \mathbf{U}_{2}^{*}\right)+\left(\mathbf{U}_{2} \otimes \boldsymbol{\Pi}_{2}^{*}\right)\right] \\
\mathbf{C}_{\Pi_{2}}= & (\mathbf{I}+\mathbf{K})\left(\boldsymbol{\Pi}_{1} \otimes \mathbf{U}_{1}\right)+\left(\mathbf{U}_{1} \otimes \boldsymbol{\Pi}_{1}\right) .
\end{aligned}
$$

Introducing these expressions in (C.4) and (C.5) gives after straightforward algebra manipulations

$$
\begin{aligned}
\mathrm{E} & {\left[\operatorname{Tr}\left(\delta \boldsymbol{\Pi}_{1, T} \mathbf{M}\right) \operatorname{Tr}\left(\delta \mathbf{\Pi}_{1, T} \mathbf{M}\right)\right] } \\
& =\frac{2}{T} \Re\left[\left(\mathbf{a}^{H} \mathbf{U}_{2} \mathbf{a}^{*}\right)\left(\mathbf{a}^{T} \mathbf{\Pi}_{2}^{*} \mathbf{a}\right)\right]+o\left(\frac{1}{T}\right) \\
\mathrm{E} & {\left[\operatorname{Tr}\left(\delta \boldsymbol{\Pi}_{2, T}^{*} \mathbf{N}\right) \operatorname{Tr}\left(\delta \boldsymbol{\Pi}_{2, T} \mathbf{N}^{*}\right)\right] } \\
& =\frac{2}{T}\left(\mathbf{a}^{H} \mathbf{U}_{1} \mathbf{a}\right)\left(\mathbf{a}^{H} \boldsymbol{\Pi}_{1} \mathbf{a}\right)+o\left(\frac{1}{T}\right) .
\end{aligned}
$$

Incorporating expressions (C.2), (C.3), (C.6), and (C.7) in (C.1) proves relation (4.4)

\section{APPENDIX D}

EXPRESSIONS OF $\mathbf{U}_{1}, \mathbf{U}_{2}, \Pi_{1}$, AND $\Pi_{2}$

To give the expressions of the blocks $\mathbf{U}_{1}, \mathbf{U}_{2}$ and $\Pi_{1}, \Pi_{2}$ from $\tilde{\mathbf{U}}=\left(\begin{array}{ll}\mathbf{U}_{1} & \mathbf{U}_{2} \\ \mathbf{U}_{2}^{*} & \mathbf{U}_{1}^{*}\end{array}\right)$ and $\tilde{\mathbf{\Pi}}=\left(\begin{array}{ll}\boldsymbol{\Pi}_{1} & \Pi_{2} \\ \Pi_{2}^{*} & \Pi_{1}^{*}\end{array}\right)$ respectively, amounts to deriving the two eigenvectors and associated eigenvalues of the rank two matrices $\tilde{\mathbf{S}}=\sigma_{x}^{2}\left(\tilde{\mathbf{a}}_{1} \tilde{\mathbf{a}}_{1}^{H}+\tilde{\mathbf{a}}_{2} \tilde{\mathbf{a}}_{2}^{H}\right)$ with $\tilde{\mathbf{a}}_{k} \stackrel{\text { def }}{=}\left(\underset{\mathbf{a}_{k}^{*} e^{-2 i \phi_{k}}}{\mathbf{a}_{k}}\right), k=1,2$.

The nonzero eigenvalues $\left(\lambda_{k}\right)_{k=1,2}$ of $\tilde{\mathbf{S}}$ are derived from their sum and product, and consequently given by the roots of the quadratic equation

$$
\lambda^{2}-\operatorname{Tr}(\tilde{\mathbf{S}}) \lambda+\operatorname{Det}\left(\sigma_{x}^{2}\left(\begin{array}{c}
\tilde{\mathbf{a}}_{1}^{H} \\
\tilde{\mathbf{a}}_{2}^{H}
\end{array}\right)\left(\tilde{\mathbf{a}}_{1}, \tilde{\mathbf{a}}_{2}\right)\right)=0
$$

which gives using $\operatorname{Tr}(\tilde{\mathbf{S}})=4 M \sigma_{x}^{2}$ and $\operatorname{Det}\left(\sigma_{x}^{2}\left(\begin{array}{l}\tilde{\mathbf{a}}_{1}^{H} \\ \tilde{\mathbf{a}}_{2}^{H}\end{array}\right)\right)$ $\left.\times\left(\tilde{\mathbf{a}}_{1}, \tilde{\mathbf{a}}_{2}\right)\right)=4 M^{2} \sigma_{x}^{4}\left(1-\varrho^{2} \cos ^{2}(\psi-\Delta \phi)\right)$, where $\varrho_{1,2}=\varrho e^{i \psi} \stackrel{\text { def }}{=}\left(\mathbf{a}_{1}^{H} \mathbf{a}_{2} / M\right)$ is the spatial complex-valued correlation coefficient between the two sources and $\Delta \phi \stackrel{\text { def }}{=} \phi_{1}-\phi_{2}$

$$
\lambda_{k}=2 M \sigma_{x}^{2}\left(1+(-1)^{k} \varrho \cos (\psi-\Delta \phi)\right), \quad k=1,2 .
$$

The associated eigenvectors $\left(\mathbf{v}_{k}\right)_{k=1,2}$ span the two-dimensional signal subspace generated by $\left(\tilde{\mathbf{a}}_{1}, \tilde{\mathbf{a}}_{2}\right)$ and therefore they are linear combinations of $\tilde{\mathbf{a}}_{1}$ and $\tilde{\mathbf{a}}_{2}$ with $\tilde{\mathbf{S}} \mathbf{v}_{k}=\lambda_{k} \mathbf{v}_{k}$, $k=1,2$. Solving this linear system, we obtain

$$
\mathbf{v}_{k}=\frac{\tilde{\mathbf{a}}_{1}+(-1)^{k} e^{-i \Delta \phi} \tilde{\mathbf{a}}_{2}}{2 \sqrt{M\left(1+(-1)^{k} \varrho \cos (\psi-\Delta \phi)\right)}}, \quad k=1,2 .
$$


With $\tilde{\mathbf{U}} \stackrel{\text { def }}{=} \sigma_{n}^{2} \tilde{\mathbf{S}}^{\#} \mathbf{R}_{\tilde{y}} \tilde{\mathbf{S}}^{\#}=\sigma_{n}^{2} \tilde{\mathbf{S}}^{\#}\left(\tilde{\mathbf{S}}+\sigma_{n}^{2} \mathbf{I}\right) \tilde{\mathbf{S}}^{\#}=$ $\sigma_{n}^{2} \tilde{\mathbf{S}}^{\#}+\sigma_{n}^{4} \tilde{\mathbf{S}} \#^{2}$ and $\tilde{\mathbf{S}}=\lambda_{1} \mathbf{v}_{1} \mathbf{v}_{1}^{H}+\lambda_{2} \mathbf{v}_{2} \mathbf{v}_{2}^{H}$, we obtain

$$
\tilde{\mathbf{U}}=\left(\frac{\sigma_{n}^{2}}{\lambda_{1}}+\frac{\sigma_{n}^{4}}{\lambda_{1}^{2}}\right) \mathbf{v}_{1} \mathbf{v}_{1}^{H}+\left(\frac{\sigma_{n}^{2}}{\lambda_{2}}+\frac{\sigma_{n}^{4}}{\lambda_{2}^{2}}\right) \mathbf{v}_{2} \mathbf{v}_{2}^{H} .
$$

Developing $\tilde{\mathbf{U}}$ w.r.t. physical parameters by using the expressions of $\left(\lambda_{k}\right)_{k=1,2}$ and $\left(\mathbf{v}_{k}\right)_{k=1,2}$ given in (D.1) and (D.2), we obtain after tedious but straightforward algebra manipulations

$$
\begin{aligned}
\mathbf{U}_{1}= & \gamma_{1}\left(\mathbf{a}_{1} \mathbf{a}_{1}^{H}+\mathbf{a}_{2} \mathbf{a}_{2}^{H}\right) \\
& +\gamma_{2}\left(e^{i \Delta \phi} \mathbf{a}_{1} \mathbf{a}_{2}^{H}+e^{-i \Delta \phi} \mathbf{a}_{2} \mathbf{a}_{1}^{H}\right) \\
\mathbf{U}_{2}= & \gamma_{1}\left(e^{2 i \phi_{1}} \mathbf{a}_{1} \mathbf{a}_{1}^{T}+e^{2 i \phi_{2}} \mathbf{a}_{2} \mathbf{a}_{2}^{T}\right) \\
& +\gamma_{2} e^{i\left(\phi_{1}+\phi_{2}\right)}\left(\mathbf{a}_{1} \mathbf{a}_{2}^{T}+\mathbf{a}_{2} \mathbf{a}_{1}^{T}\right)
\end{aligned}
$$

where $\gamma_{1}$ and $\gamma_{2}$ are the following ASNR (defined by $r \stackrel{\text { def }}{=}$ $M \sigma_{x}^{2} / \sigma_{n}^{2}$ ) dependent geometric terms

$$
\begin{aligned}
\gamma_{1}= & \frac{1}{4 r M\left(1-\varrho^{2} \cos ^{2}(\psi-\Delta \phi)\right)} \\
& \times\left(1+\varrho^{2} \cos ^{2}(\psi-\Delta \phi)+\frac{1+3 \varrho^{2} \cos ^{2}(\psi-\Delta \phi)}{2 r\left(1-\varrho^{2} \cos ^{2}(\psi-\Delta \phi)\right)}\right) \\
\gamma_{2}= & \frac{-\varrho \cos (\psi-\Delta \phi))}{2 r M\left(1-\varrho^{2} \cos ^{2}(\psi-\Delta \phi)\right)^{2}} \\
& \times\left(1+\frac{3+\varrho^{2} \cos ^{2}(\psi-\Delta \phi)}{4 r\left(1-\varrho^{2} \cos ^{2}(\psi-\Delta \phi)\right)}\right) .
\end{aligned}
$$

Finally, $\Pi_{1}$ and $\Pi_{2}$ are deduced from $\tilde{\Pi}=\mathbf{I}-\left(\mathbf{v}_{1} \mathbf{v}_{1}^{H}+\mathbf{v}_{2} \mathbf{v}_{2}^{H}\right)$ and the expressions of $\mathbf{v}_{1}$ and $\mathbf{v}_{2}$ given in (D.2). After tedious but straightforward algebra manipulations, we obtain

$$
\begin{aligned}
\Pi_{1}= & \mathbf{I}+\tau_{1}\left(\mathbf{a}_{1} \mathbf{a}_{1}^{H}+\mathbf{a}_{2} \mathbf{a}_{2}^{H}\right) \\
& +\tau_{2}\left(e^{i \Delta \phi} \mathbf{a}_{1} \mathbf{a}_{2}^{H}+e^{-i \Delta \phi} \mathbf{a}_{2} \mathbf{a}_{1}^{H}\right) \\
\Pi_{2}= & \tau_{1}\left(e^{2 i \phi_{1}} \mathbf{a}_{1} \mathbf{a}_{1}^{T}+e^{2 i \phi_{2}} \mathbf{a}_{2} \mathbf{a}_{2}^{T}\right) \\
& +\tau_{2} e^{i\left(\phi_{1}+\phi_{2}\right)}\left(\mathbf{a}_{1} \mathbf{a}_{2}^{T}+\mathbf{a}_{2} \mathbf{a}_{1}^{T}\right)
\end{aligned}
$$

where $\tau_{1}$ and $\tau_{2}$ are the following purely geometric terms:

$$
\begin{aligned}
\tau_{1} & =-\frac{1}{2 M\left(1-\varrho^{2} \cos ^{2}(\psi-\Delta \phi)\right)} \\
\tau_{2} & =-\tau_{1} \varrho \cos (\psi-\Delta \phi) .
\end{aligned}
$$

\section{APPENDIX E}

\section{PROOF OF RESULT 2}

For simplifying the notations to derive the two expressions of the threshold ASNR $\xi_{1}$ and $\xi_{2}$ given by the Cox and the Sharman and Durrani criteria respectively, we first consider centrosymmetric arrays. ${ }^{6}$ This assumption ${ }^{7}$ that means that the steering vectors $\mathbf{a}(\theta)$ satisfy $\mathbf{J a}(\theta)=\mathbf{a}^{*}(\theta)$, implies that the different

\footnotetext{
${ }^{6}$ We note that this structure is very used in practice because uniform linear, uniform circular, and regular hexagonal shaped arrays [26] are centrosymmetric.

${ }^{7}$ We note that this assumption necessarily implies that the origin of the coordinate system is at the array centroid.
}

spatial correlation coefficients: $\varrho_{1,2}, \varrho_{k, m} \stackrel{\text { def }}{=} \mathbf{a}_{k}^{H} \mathbf{a}_{m} / M, k=$ 1,2 with $\mathbf{a}_{m} \stackrel{\text { def }}{=} \mathbf{a}\left(\theta_{m}\right), \varrho_{k, m}^{\prime} \stackrel{\text { def }}{=} \mathbf{a}_{k}^{H} \mathbf{a}_{m}^{\prime} / M, k=1,2$ with $\left.\mathbf{a}_{m}^{\prime} \stackrel{\text { def }}{=}(d \mathbf{a}(\theta) / d \theta)\right|_{\theta=\theta_{m}}$ and $\varrho_{k, m}^{\prime \prime} \stackrel{\text { def }}{=} \mathbf{a}_{k}^{H} \mathbf{a}_{m}^{\prime \prime} / M, k=1,2$ with $\left.\mathbf{a}_{m}^{\prime \prime} \stackrel{\text { def }}{=}\left(d^{2} \mathbf{a}(\theta)^{2} / d \theta\right)\right|_{\theta=\theta_{m}}$ that will be used in the proof are real-valued.

Consider the Cox criterion. From (4.4)

$$
\begin{aligned}
& \mathrm{E}\left(g_{T}^{\mathrm{Alg}_{\mathrm{NC}}}\left(\theta_{1}\right)\right)=\frac{2}{T}(2 M-3)\left[\left(\mathbf{a}_{1}^{H} \mathbf{U}_{1} \mathbf{a}_{1}\right)\left(\mathbf{a}_{1}^{H} \mathbf{\Pi}_{1} \mathbf{a}_{1}\right)\right. \\
& \left.-\Re\left[\left(\mathbf{a}_{1}^{H} \mathbf{U}_{2} \mathbf{a}_{1}^{*}\right)\left(\mathbf{a}_{1}^{T} \mathbf{\Pi}_{2}^{*} \mathbf{a}_{1}\right)\right]\right]+o\left(\frac{1}{T}\right) .
\end{aligned}
$$

Expressing $\mathbf{a}_{1}^{H} \mathbf{U}_{1} \mathbf{a}_{1}$ and $\mathbf{a}_{1}^{H} \mathbf{U}_{2} \mathbf{a}_{1}^{*}$ as function of the ASNR $r$ using (D.3) and (D.4) respectively, and also $\mathbf{a}_{1}^{H} \boldsymbol{\Pi}_{1} \mathbf{a}_{1}$ and $\mathbf{a}_{1}^{T} \Pi_{2}^{*} \mathbf{a}_{1}$ as function of the spatial correlation coefficient $\varrho_{1,2}$, using (D.5) and (D.6), respectively, we obtain after tedious but straightforward algebraic manipulations

$$
\begin{array}{r}
\mathrm{E}\left(g_{T}^{\mathrm{Alg}_{\mathrm{NC}}}\left(\theta_{1}\right)\right)=\frac{1}{T}\left(\frac{1}{r} h_{1}\left(\varrho_{1,2}, \Delta \phi\right)+\frac{1}{r^{2}}\right. \\
\left.h_{2}\left(\varrho_{1,2}, \Delta \phi\right)\right) \\
+o\left(\frac{1}{T}\right)
\end{array}
$$

with

$$
\begin{aligned}
& h_{1}\left(\varrho_{1,2}, \Delta \phi\right)=\frac{(2 M-3) M^{2}\left(1-\varrho_{1,2}^{2}\right)}{2\left(1-\varrho_{1,2}^{2} \cos ^{2}(\Delta \phi)\right)} \\
& h_{2}\left(\varrho_{1,2}, \Delta \phi\right)=\frac{h_{1}\left(\varrho_{1,2}, \Delta \phi\right)}{2\left(1-\varrho_{1,2}^{2} \cos ^{2}(\Delta \phi)\right)} .
\end{aligned}
$$

To derive $\mathrm{E}\left(g_{T}^{\mathrm{Alg}}{ }_{\mathrm{NC}}\left(\theta_{m}\right)\right), \mathbf{a}_{m}^{H} \mathbf{U}_{1} \mathbf{a}_{m}$ and $\mathbf{a}_{m}^{H} \mathbf{U}_{2} \mathbf{a}_{m}^{*}$ are expressed as function of the ASNR $r$ using (D.3) and (D.4) respectively, and also $\mathbf{a}_{m}^{H} \boldsymbol{\Pi}_{1} \mathbf{a}_{m}$ and $\mathbf{a}_{m}^{T} \boldsymbol{\Pi}_{2}^{*} \mathbf{a}_{m}$ as function of $\varrho_{1, m}$ and $\varrho_{2, m}$ using (D.5) and (D.6), respectively, we obtain after tedious but straightforward algebraic manipulations

$$
\begin{aligned}
\mathrm{E}\left(g_{T}^{\operatorname{Alg}_{\mathrm{NC}}}\left(\theta_{m}\right)=\right. & g^{\mathrm{Alg}_{\mathrm{NC}}}\left(\theta_{m}\right) \\
& +\frac{1}{T}\left(\frac{1}{r} h_{1, m}+\frac{1}{r^{2}} h_{2, m}\right)+o\left(\frac{1}{T}\right)
\end{aligned}
$$

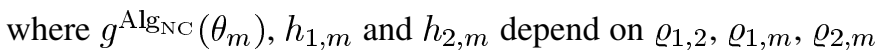
and $\Delta \phi$, and are given by

$$
\begin{aligned}
& g^{\operatorname{Alg}_{\mathrm{NC}}\left(\theta_{m}\right)} \\
& =\frac{M^{2}}{1-\varrho_{1,2}^{2} \cos ^{2}(\Delta \phi)} \\
& \times\left(1-\varrho_{1,2}^{2}-\varrho_{1, m}^{2}-\varrho_{2, m}^{2}+2 \varrho_{1,2} \varrho_{1, m} \varrho_{2, m}+\sin ^{2}(\Delta \phi)\right. \\
& \left.\times\left(\varrho_{1, m} \varrho_{2, m}\left(\varrho_{1, m} \varrho_{2, m}-2 \varrho_{1,2}\right)+\varrho_{1,2}^{2}\right)\right) \\
& h_{1, m} \\
& =\frac{2 g^{\operatorname{Alg}_{\mathrm{NC}}\left(\theta_{m}\right)+(2 M-3) \eta_{1}}}{1-\varrho_{1,2}^{2} \cos ^{2}(\Delta \phi)} \\
& h_{2, m} \\
& =\frac{g^{\operatorname{Alg}_{\mathrm{NC}}}\left(\theta_{m}\right)+(2 M-3) \eta_{2}}{1-\varrho_{1,2}^{2} \cos ^{2}(\Delta \phi)}
\end{aligned}
$$


where

$$
\begin{aligned}
\eta_{1}= & \frac{M^{2}}{2\left(1-\varrho_{1,2}^{2} \cos ^{2}(\Delta \phi)\right)} \\
& \times\left(\operatorname { c o s } ^ { 2 } ( \Delta \phi ) \left(2 \varrho_{1, m} \varrho_{2, m}\left(2 \varrho_{1,2}-\varrho_{1, m} \varrho_{2, m}\right)\right.\right. \\
& \left.\quad-\varrho_{1,2}^{2}\left(\varrho_{1, m}^{2}+\varrho_{2, m}^{2}\right)\right) \\
& \left.\quad-\varrho_{1, m}^{2}-\varrho_{2, m}^{2}+2 \varrho_{1, m} \varrho_{2, m}\right) \\
\eta_{2}= & \frac{M^{2}}{4\left(1-\varrho_{1,2}^{2} \cos ^{2}(\Delta \phi)\right)} \\
& \times\left(\left(1+\varrho_{1,2}^{2} \cos ^{2}(\Delta \phi)\right)\left(2 \varrho_{1, m}^{2} \varrho_{2, m}^{2}-\varrho_{1, m}^{2}-\varrho_{2, m}^{2}\right)\right. \\
& \quad+2 \cos ^{2}(\Delta \phi)\left(2 \varrho_{1,2} \varrho_{1, m} \varrho_{2, m}-\varrho_{1,2}^{2}\left(\varrho_{1, m}^{2}+\varrho_{2, m}^{2}\right)\right. \\
& \quad+\varrho_{1, m} \varrho_{2, m}\left(\varrho_{1,2}-\varrho_{1, m} \varrho_{2, m}\right) \\
& \left.\left.\times\left(1+\varrho_{1,2} \cos (\Delta \phi)\right)\right)\right) .
\end{aligned}
$$

Finally, the threshold ASNR given by the Cox criterion is deduced from the value $\xi_{1}$ of $r$ for which expressions (E.1) and (E.2) are equal

$$
\xi_{1}=\frac{1}{T} \alpha_{1}\left(1+\sqrt{1+\frac{T}{\beta_{1}}}\right)
$$

with $\alpha_{1} \stackrel{\text { def }}{=}\left(h_{1}-h_{1, m}\right) / 2 g^{\operatorname{Alg}_{\mathrm{NC}}}\left(\theta_{m}\right)$ and $\beta_{1} \stackrel{\text { def }}{=}\left(h_{1}-\right.$ $\left.h_{1, m}\right)^{2} / 4 g^{\operatorname{Alg}_{\mathrm{NC}}}\left(\theta_{m}\right)\left(h_{2}-h_{2, m}\right)$ which depend on $\Delta \theta, \Delta \phi, M$ and the specific array. Using expansions of the spatial correlation coefficients $\varrho_{1,2}, \varrho_{1, m}$ and $\varrho_{2, m}$ w.r.t. $\Delta \theta$ for closely spaced sources and a symbolic calculus akin to a high level language to achieve the algebra manipulations, completes the proof of (4.5). For example, for a ULA, using the parametrization recalled at the end of Section III

$$
\varrho_{1,2}=\frac{\sin \left(M \frac{\Delta \theta}{2}\right)}{M \sin \left(\frac{\Delta \theta}{2}\right)} \text { and } \varrho_{1, m}=\varrho_{2, m}=\frac{\sin \left(M \frac{\Delta \theta}{4}\right)}{M \sin \left(\frac{\Delta \theta}{4}\right)}
$$

with

$$
\begin{aligned}
\frac{\sin \left(M \frac{\Delta \theta}{2}\right)}{M \sin \left(\frac{\Delta \theta}{2}\right)}=1- & \frac{\left(M^{2}-1\right)(\Delta \theta)^{2}}{24} \\
& +\frac{\left(M^{2}-1\right)\left(3 M^{2}-7\right)(\Delta \theta)^{4}}{5760}+O\left((\Delta \theta)^{6}\right) .
\end{aligned}
$$

To consider now the Sharman and Durrani criterion for which the threshold ASNR $\xi_{2}$ is deduced from nulling the second derivative of the mean null spectrum at the midpoint $\theta_{m}$ (see (3.4)), we follow the same steps used for deriving the threshold ASNR associated with the conventional MUSIC algorithm detailed in [10]. The main steps are given in the following in which the mean null spectrum given by (4.4) is written for $K=2$ as

$$
\begin{aligned}
\mathrm{E}\left(g_{T}^{\mathrm{Alg}_{\mathrm{NC}}}(\theta)\right)=\left(1-\frac{4}{T}\right. & \left.\operatorname{Tr}\left(\mathbf{U}_{1}\right)\right) g^{\mathrm{Alg}_{\mathrm{NC}}(\theta)} \\
& -\frac{2(2 M-3)}{T} f(\theta)+o\left(\frac{1}{T}\right)
\end{aligned}
$$

with

$$
f(\theta) \stackrel{\text { def }}{=} \Re\left(\operatorname{Tr}\left(\mathbf{U}_{2} \mathbf{N}^{*}\right) \operatorname{Tr}\left(\mathbf{\Pi}_{2}^{*} \mathbf{N}\right)\right)-\operatorname{Tr}\left(\mathbf{\Pi}_{1} \mathbf{M}\right) \operatorname{Tr}\left(\mathbf{U}_{1} \mathbf{M}\right) .
$$

Differentiating (E.3) twice w.r.t. $\theta$, leads to

$$
\begin{aligned}
& \left.\frac{d^{2} \mathrm{E}\left(g_{T}^{\mathrm{Alg}_{\mathrm{NC}}}(\theta)\right)}{d \theta^{2}}\right|_{\theta=\theta_{m}} \\
& =\left.\left(1-\frac{4}{T} \operatorname{Tr}\left(\mathbf{U}_{1}\right)\right) \frac{d^{2} g^{\mathrm{Alg}_{\mathrm{NC}}(\theta)}}{d \theta^{2}}\right|_{\theta=\theta_{m}} \\
& \quad-\left.\frac{2(2 M-3)}{T} \frac{d^{2} f(\theta)}{d \theta^{2}}\right|_{\theta=\theta_{m}}+o\left(\frac{1}{T}\right),
\end{aligned}
$$

with

$$
\begin{aligned}
& \left.\frac{d^{2} g^{\operatorname{Alg}_{N C}(\theta)}}{d \theta^{2}}\right|_{\theta=\theta_{m}} \\
& =2\left(\left(\operatorname{Tr}\left(\mathbf{\Pi}_{1} \mathbf{M}_{m}^{\prime}\right)\right)^{2}+\operatorname{Tr}\left(\mathbf{\Pi}_{1} \mathbf{M}_{m}\right) \operatorname{Tr}\left(\mathbf{\Pi}_{1} \mathbf{M}_{m}^{\prime \prime}\right)\right. \\
& \left.-\left|\operatorname{Tr}\left(\boldsymbol{\Pi}_{2} \mathbf{N}_{m}^{\prime *}\right)\right|^{2}-\Re\left(\operatorname{Tr}\left(\mathbf{\Pi}_{2} \mathbf{N}_{m}^{*}\right) \operatorname{Tr}\left(\boldsymbol{\Pi}_{2}^{*} \mathbf{N}_{m}^{\prime \prime}\right)\right)\right) \\
& \left.\frac{d^{2} f(\theta)}{d \theta^{2}}\right|_{\theta=\theta_{m}} \\
& =\Re\left(2 \operatorname{Tr}\left(\mathbf{U}_{2} \mathbf{N}_{m}^{\prime *}\right) \operatorname{Tr}\left(\Pi_{2}^{*} \mathbf{N}_{m}^{\prime}\right)\right. \\
& \left.+\operatorname{Tr}\left(\mathbf{U}_{2} \mathbf{N}_{m}^{\prime \prime *}\right) \operatorname{Tr}\left(\boldsymbol{\Pi}_{2}^{*} \mathbf{N}_{m}\right)+\operatorname{Tr}\left(\mathbf{U}_{2} \mathbf{N}_{m}^{*}\right) \operatorname{Tr}\left(\boldsymbol{\Pi}_{2}^{*} \mathbf{N}_{m}^{\prime \prime}\right)\right) \\
& -2 \operatorname{Tr}\left(\Pi_{1} \mathbf{M}_{m}^{\prime}\right) \operatorname{Tr}\left(\mathbf{U}_{1} \mathbf{M}_{m}^{\prime}\right)-\operatorname{Tr}\left(\boldsymbol{\Pi}_{1} \mathbf{M}_{m}^{\prime \prime}\right) \operatorname{Tr}\left(\mathbf{U}_{1} \mathbf{M}_{m}\right) \\
& -\operatorname{Tr}\left(\mathbf{\Pi}_{1} \mathbf{M}_{m}\right) \operatorname{Tr}\left(\mathbf{U}_{1} \mathbf{M}_{m}^{\prime \prime}\right) \text {, }
\end{aligned}
$$

where $\mathbf{M}_{m} \stackrel{\text { def }}{=} \mathbf{M}\left(\theta_{m}\right), \mathbf{N}_{m} \stackrel{\text { def }}{=} \mathbf{N}\left(\theta_{m}\right), \mathbf{M}_{m}^{\prime} \stackrel{\text { def }}{=}$ $\left.(d \mathbf{M}(\theta) / d \theta)\right|_{\theta=\theta_{m}},\left.\quad \mathbf{N}_{m}^{\prime} \stackrel{\text { def }}{=}(d \mathbf{N}(\theta) / d \theta)\right|_{\theta=\theta_{m}}, \mathbf{M}_{m}^{\prime \prime} \stackrel{\text { def }}{=}$ $\left.\left(d^{2} \mathbf{M}(\theta) / d \theta^{2}\right)\right|_{\theta=\theta_{m}}$ and $\left.\mathbf{N}_{m}^{\prime \prime} \stackrel{\text { def }}{=}\left(d^{2} \mathbf{N}(\theta) / d \theta^{2}\right)\right|_{\theta=\theta_{m}}$. By expressing $\operatorname{Tr}\left(\mathbf{U}_{1} \mathbf{M}_{m}\right), \operatorname{Tr}\left(\mathbf{U}_{1} \mathbf{M}_{m}^{\prime}\right)$ and $\operatorname{Tr}\left(\mathbf{U}_{1} \mathbf{M}_{m}^{\prime \prime}\right)$, and $\operatorname{Tr}\left(\mathbf{U}_{2} \mathbf{N}_{m}^{*}\right), \operatorname{Tr}\left(\mathbf{U}_{2} \mathbf{N}_{m}^{\prime *}\right)$ and $\operatorname{Tr}\left(\mathbf{U}_{2} \mathbf{M}_{1, m}^{\prime \prime *}\right)$ as a function of the ASNR $r$ using (D.3) and (D.4), respectively, and expressing $\operatorname{Tr}\left(\boldsymbol{\Pi}_{1} \mathbf{M}_{m}\right), \operatorname{Tr}\left(\boldsymbol{\Pi}_{1} \mathbf{M}_{m}^{\prime}\right)$ and $\operatorname{Tr}\left(\boldsymbol{\Pi}_{1} \mathbf{M}_{m}^{\prime \prime}\right)$, and $\operatorname{Tr}\left(\boldsymbol{\Pi}_{2} \mathbf{N}_{m}^{*}\right)$, $\operatorname{Tr}\left(\boldsymbol{\Pi}_{2} \mathbf{N}_{m}^{\prime *}\right)$ and $\operatorname{Tr}\left(\boldsymbol{\Pi}_{2} \mathbf{N}_{m}^{\prime \prime *}\right)$ as a function of $\varrho_{k, m}^{\prime}$ and $\varrho_{k, m}^{\prime \prime}$, using (D.5) and (D.6), respectively, we obtain after tedious but straightforward algebraic manipulations

$$
\left.\frac{d^{2} f(\theta)}{d \theta^{2}}\right|_{\theta=\theta_{m}}=\frac{\nu_{1}}{r}+\frac{\nu_{2}}{r^{2}}
$$

with

$$
\begin{aligned}
\nu_{1} \stackrel{\text { def }}{=} & \frac{M^{2}}{2\left(1-\varrho_{1,2}^{2} \cos ^{2}(\Delta \phi)\right)^{2}} \\
& \times\left(8 M^{2} \sin ^{2}(\Delta \phi)\right. \\
& \quad \times\left(\left(\varrho_{1, m} \varrho_{2, m}^{\prime}+\varrho_{1, m}^{\prime} \varrho_{2, m}\right)^{2}+\varrho_{1, m} \varrho_{2, m} k_{1}\right)-k_{2} \\
& \left.\quad-\varrho_{1,2} \cos ^{2}(\Delta \phi)\left(\varrho_{1,2} k_{2}-2 k_{1}\right)\right)
\end{aligned}
$$


where $k_{1} \stackrel{\text { def }}{=} \varrho_{1, m} \varrho_{2, m}^{\prime \prime}+\varrho_{1, m}^{\prime \prime} \varrho_{2, m}+2 \varrho_{1, m}^{\prime} \varrho_{2, m}^{\prime}$ and $k_{2} \stackrel{\text { def }}{=}$ $\varrho_{1, m}^{\prime 2}+\varrho_{2, m}^{\prime 2}+\varrho_{1, m} \varrho_{1, m}^{\prime \prime}+\varrho_{2, m} \varrho_{2, m}^{\prime \prime}$ and where the spatial correlation coefficients $\varrho_{k, m}^{\prime}, \varrho_{k, m}^{\prime \prime}, k=1,2$ depend on $\Delta \theta$, $M$ and the specific array. From the expression of $\mathbf{U}_{1}$ given by (D.3), where here $\varrho_{1,2}$ is real-valued and consequently $\psi=0$, we obtain

$$
\begin{aligned}
\operatorname{Tr}\left(\mathbf{U}_{1}\right)=\frac{1}{2\left(1-\varrho_{1,2}^{2} \cos ^{2}(\Delta \phi)\right)} & \frac{1}{r} \\
& +\frac{\left(1+\varrho_{1,2}^{2} \cos ^{2}(\Delta \phi)\right)}{4\left(1-\varrho_{1,2}^{2} \cos ^{2}(\Delta \phi)\right)^{2}} \frac{1}{r^{2}} .
\end{aligned}
$$

Inserting (E.6) and (E.5) into (E.4), we obtain after straightforward algebraic manipulations

$$
\begin{aligned}
\left.\frac{d^{2} \mathrm{E}\left(g_{T}^{\mathrm{Alg}_{\mathrm{NC}}}(\theta)\right)}{d \theta^{2}}\right|_{\theta=\theta_{m}}= & \left.\frac{d^{2} g^{\mathrm{Alg}_{\mathrm{NC}}}(\theta)}{d \theta^{2}}\right|_{\theta=\theta_{m}} \\
& -\frac{1}{T}\left[\frac{1}{r} h_{1, m}^{\prime}+\frac{1}{r^{2}} h_{2, m}^{\prime}\right]
\end{aligned}
$$

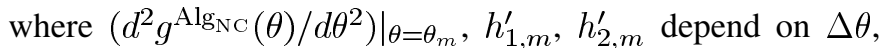
$\Delta \phi, M$ and the specific array are given by the equation shown at the bottom of the page. Finally, the threshold ASNR given by the Sharman and Durrani criterion is deduced from the value $\xi_{2}$ of $r$ for which expression (E.7) of the second derivative of the mean null spectrum at the midpoint $\theta_{m}$ is null

$$
\xi_{2}=\frac{1}{T} \alpha_{2}\left(1+\sqrt{1+\frac{T}{\beta_{2}}}\right)
$$

with $\alpha_{2} \stackrel{\text { def }}{=} h_{1, m}^{\prime} / 2 h_{m}$ and $\beta_{2} \stackrel{\text { def }}{=} h_{1, m}^{\prime 2} / 4 h_{m} h_{2, m}^{\prime}$ which depend on $\Delta \theta, \Delta \phi, M$ and the specific array. Using expansions of the spatial correlation coefficients $\varrho_{1,2}, \varrho_{1, m}, \varrho_{2, m}$ and of $\varrho_{1, m}^{\prime}, \varrho_{2, m}^{\prime} \varrho_{1, m}^{\prime \prime}, \varrho_{2, m}^{\prime \prime}$ w.r.t. $\Delta \theta$ for closely spaced sources and a symbolic calculus akin to a high level language to achieve the algebra manipulations, completes the proof of (4.6). For example, for a ULA using the parametrization recalled at the end of Section III

$$
\begin{aligned}
\varrho_{1, m}^{\prime}= & -\varrho_{2, m}^{\prime} \\
= & \frac{M \cos \left(M \frac{\Delta \theta}{4}\right) \sin \left(\frac{\Delta \theta}{4}\right)-\cos \left(\frac{\Delta \theta}{4}\right) \sin \left(M \frac{\Delta \theta}{4}\right)}{2 M \sin ^{2}\left(\frac{\Delta \theta}{4}\right)} \\
= & -\frac{\left(M^{2}-1\right)(\Delta \theta)}{24}+\frac{\left(M^{2}-1\right)\left(3 M^{2}-7\right)(\Delta \theta)^{3}}{11520} \\
& +O\left((\Delta \theta)^{5}\right)
\end{aligned}
$$

and

$$
\begin{aligned}
\varrho_{1, m}^{\prime \prime}= & -\varrho_{2, m}^{\prime \prime} \\
= & \frac{1}{4 M \sin ^{3}\left(\frac{\Delta \theta}{4}\right)}\left(\sin \left(M \frac{\Delta \theta}{4}\right)\left(2-\left(1+M^{2}\right) \sin ^{2}\left(\frac{\Delta \theta}{4}\right)\right)\right. \\
& \left.-M \cos \left(M \frac{\Delta \theta}{4}\right) \sin \left(\frac{\Delta \theta}{2}\right)\right) \\
= & -\frac{M^{2}-1}{12}+\frac{\left(M^{2}-1\right)\left(3 M^{2}-7\right)(\Delta \theta)^{2}}{1920} \\
& -\frac{\left(M^{2}-1\right)\left(3 M^{4}-18 M^{2}+31\right)(\Delta \theta)^{4}}{516096}+O\left((\Delta \theta)^{5}\right) .
\end{aligned}
$$

To extend the proof to an arbitrary array, it is convenient to use the general parametrization proposed by Lee et al. [6] that has been recalled at the end of Section IV where $\Delta \theta$ is defined here by $\Delta \theta=\theta_{1}-\theta_{2}$ rather than $\Delta \theta=(2 \pi / \lambda) \sqrt{(1 / M) \sum_{m=1}^{M} \mathbf{r}_{m}^{T}\left(\mathbf{i}\left(\theta_{1}\right)-\mathbf{i}\left(\theta_{2}\right)\right)}[6$, rel. (8)]. In this case, all the steps of the previous proof extend, but now the spatial correlation coefficients $\varrho_{1,2}, \varrho_{k, m} \varrho_{k, m}^{\prime}$ and $\varrho_{k, m}^{\prime \prime}$, $k=1,2$ are generally complex-valued and consequently the notation are much cumbersome and furthermore the expansions of the spatial correlation coefficients are much more involved. For example, in the derivation of $\varrho_{1,2}$ and $\varrho_{1, m}$, we obtain

$$
\begin{aligned}
\varrho_{1,2} & =\frac{1}{M} \sum_{m=1}^{M} e^{i \frac{4 \pi}{\lambda} \mathbf{r}_{m}^{T} \mathbf{i}_{0} \sin \left(\frac{\Delta \theta}{2}\right)} \text { and } \\
\varrho_{1, m} & =\frac{1}{M} \sum_{m=1}^{M} e^{i \frac{2 \pi}{\lambda} \mathbf{r}_{m}^{T}\left(\left(\cos \left(\frac{\Delta \theta}{2}\right)-1\right) \mathbf{i}_{m}+\sin \left(\frac{\Delta \theta}{2}\right) \mathbf{i}_{0}\right)}
\end{aligned}
$$

where $\mathbf{i}_{0}$ is the unit vector parallel to $\mathbf{i}_{1}-\mathbf{i}_{2}$ and $\mathbf{i}_{m}$ is the bisector of $\mathbf{i}_{1}$ and $\mathbf{i}_{2}$, which gives the expansions

$$
\begin{gathered}
\varrho_{1,2}=1-\frac{1}{M} \sum_{m=1}^{M}\left(\frac{a_{m}^{2}}{2}(\Delta \theta)^{2}+i \frac{a_{m}^{3}}{6}(\Delta \theta)^{3}\right. \\
\left.\quad-\frac{a_{m}^{4}+a_{m}^{2}}{24}(\Delta \theta)^{4}\right)+O\left((\Delta \theta)^{5}\right) \\
\varrho_{1, m}=1-\frac{1}{M} \sum_{m=1}^{M}\left(\frac{1}{8} a_{m}^{2}(\Delta \theta)^{2}+\frac{1}{48} a_{m}\left(i a_{m}^{2}-3 b_{m}\right)(\Delta \theta)^{3}\right. \\
\left.\quad-\frac{1}{384}\left(4 a_{m}^{2}-3 b_{m}^{2}+6 i b_{m} a_{m}^{2}+a_{m}^{4}\right)(\Delta \theta)^{4}\right) \\
+O\left((\Delta \theta)^{5}\right) \\
\text { with } a_{m} \stackrel{\text { def }}{=}(2 \pi / \lambda) \mathbf{r}_{m}^{T} \mathbf{i}_{0} \text { and } b_{m} \stackrel{\text { def }}{=}(2 \pi / \lambda) \mathbf{r}_{m}^{T} \mathbf{i}_{m} .
\end{gathered}
$$

$$
\begin{aligned}
& \left.\frac{d^{2} g^{\mathrm{Alg}}{ }_{\mathrm{NC}}(\theta)}{d \theta^{2}}\right|_{\theta=\theta_{m}}=\frac{2 M^{2}}{\left(1-\varrho_{1,2}^{2} \cos ^{2}(\Delta \phi)\right)^{2}}\left(\left(1-\varrho_{1,2}^{2} \cos ^{2}(\Delta \phi)\right)\left(\varrho_{1,2} k_{1} \cos ^{2}(\Delta \phi)-k_{2}\right)+\sin ^{2}(\Delta \phi)\right. \\
& \times\left(\left(1-\varrho_{1,2}^{2} \cos ^{2}(\Delta \phi)\right)\left(\varrho_{1, m} \varrho_{2, m}^{\prime}+\varrho_{1, m}^{\prime} \varrho_{2, m}\right)\right. \\
& \left.\left.+k_{3}-\varrho_{1,2}^{2} \varrho_{1, m} \varrho_{2, m} k_{1} \cos ^{2}(\Delta \phi)\right)\right) \stackrel{\text { def }}{=} h_{m} \\
& h_{1, m}^{\prime} \stackrel{\text { def }}{=} \frac{2\left(h_{m}+(2 M-3) \nu_{1}\left(1-\varrho_{1,2}^{2} \cos ^{2}(\Delta \phi)\right)\right)}{1-\varrho_{1,2}^{2} \cos ^{2}(\Delta \phi)} \\
& h_{2, m}^{\prime} \stackrel{\text { def }}{=} \frac{h_{m}\left(1+\varrho_{1,2}^{2} \cos ^{2}(\Delta \phi)\right)+2(2 M-3) \nu_{2}\left(1-\varrho_{1,2}^{2} \cos ^{2}(\Delta \phi)\right)^{2}}{\left(1-\varrho_{1,2}^{2} \cos ^{2}(\Delta \phi)\right)^{2}} .
\end{aligned}
$$




\section{REFERENCES}

[1] B. D. Steinberg, Principles of Aperture of Array System Design. New York: Wiley, 1976.

[2] H. Cox, "Resolving power and sensitivity to mismatch of optimum array processors," J. Acoust. Soc. Amer., vol. 54, no. 3, pp. 771-785, 1973.

[3] M. Kaveh and A. J. Barabell, "The statistical performance of the MUSIC and the Minimum-Norm algorithms in resolving plane waves in noise," IEEE Trans. Acoust., Speech, Signal Process., vol. 34, no. 2, pp. 331-341, Apr. 1986.

[4] M. Kaveh and H. Wang, "Threshold properties of narrowband signal subspace array processing methods," in Advances in Spectrum Analysis and Array Processing, S. Haykin, Ed. Englewood Cliffs, NJ: Prentice-Hall, 1991, vol. 2, pp. 173-220.

[5] S. U. Pillai and G. H. Kwon, "Performance analysis of MUSIC-type high resolution estimators for direction finding in correlated and coherent scenes," IEEE Trans. Acoust., Speech, Signal Process., vol. 37, no. 8, pp. 1176-1189, Aug. 1989.

[6] H. B. Lee and M. S. Wengrovitz, "Resolution threshold of beamspace MUSIC for two closely spaced emitters," IEEE Trans. Signal Process., vol. 38, no. 9, pp. 1445-1559, Sep. 1990.

[7] C. Zhou, F. Haber, and D. L. Jaggard, "A resolution measure for the MUSIC algorithm and its application to plane wave arrivals contaminated by coherent interference," IEEE Trans. Signal Process., vol. 39, no. 2, pp. 454-463, Feb. 1991.

[8] K. C. Sharman and S. T. Durrani, "Resolving power of signal subspace methods for finite data lengths," presented at the Int. Conf. Acoustics, Speech, Signal Processing (ICASSP), Tampa, Florida, Apr. 1985.

[9] P. Foster and E. Villier, "Simplified formulas for performance analysis of MUSIC and Min Norm," presented at the OCEANS'98, Nice, France, Sep. 1998.

[10] P. Foster and E. Villier, "Performances Asymptotiques des Méthodes Haute Résolution," in Les Méthodes à Haute Résolution, S. Marcos, Ed. Paris, France: Hermes, 1998.

[11] H. B. Lee and M. S. Wengrovitz, "Statistical characterization of the MUSIC algorithm null spectrum," IEEE Trans. Acoust., Speech, Signal Process., vol. 39, no. 6, pp. 1333-1347, Jun. 1991.

[12] Q. T. Zhang, "Probability of resolution of the MUSIC algorithm," IEEE Trans. Signal Process., vol. 43, no. 4, pp. 978-987, Apr. 1995.

[13] Q. T. Zhang, "A statistical resolution theory of the beamformer-based spatial spectrum for determining the directions of signals in white noise," IEEE Trans. Acoust., Speech, Signal Process., vol. 43, no. 8, pp. 1867-1873, Aug. 1995

[14] S. T. Smith, "Statistical resolution limits and the complexified Cramer-Rao bound," IEEE Trans. Signal Process., vol. 53, no. 5, pp. 1597-1609, May 2005.

[15] J. P. Delmas and H. Abeida, "Statistical resolution limits of DOA for discrete sources," presented at the Int. Conf. Acoustics, Speech, Signal Processing (ICASSP), Toulouse, May 2006.

[16] Z. Lui and A. Nehorai, "Statistical angular resolution limit for point sources," IEEE Trans. Signal Process., vol. 55, no. 11, pp. 5521-5527, Nov. 2007.

[17] H. Abeida and J. P. Delmas, "MUSIC-like estimation of direction of arrival for non-circular sources," IEEE Trans. Signal Process., vol. 54, no. 7, pp. 2678-2690, Jul. 2006.

[18] P. Chargé, Y. Wang, and J. Saillard, "A non-circular sources direction finding method using polynomial rooting," Signal Process., vol. 81, pp. 1765-1770, 2001
[19] H. Krim, P. Forster, and G. Proakis, "Operator approach to performance analysis of root-MUSIC and root-min-norm," IEEE Trans. Signal Process., vol. 40, no. 7, pp. 1687-1696, Jul. 1992.

[20] X. Mestre and F. Rubio, "An improved subspace-based algorithm in the small sample size regime," presented at the Int. Conf. Acoustics, Speech, Signal Processing (ICASSP), Toulouse, France, May 2006.

[21] J. P. Delmas and H. Abeida, "Stochastic Cramer-Rao bound for noncircular signals with application to DOA estimation," Trans. Signal Process., vol. 52, no. 11, pp. 3192-3199, Nov. 2004

[22] D. Rife and R. Boorstyn, "Single tone parameter estimation from discrete-time observations," Trans. Inf. Theory, vol. 20, no. 5, pp. 591-598, Sep. 1974.

[23] H. L. Van Trees, Detection, Estimation and Modulation Theory. New York: Wiley, 1968, pt. 1.

[24] J. R. Schott, Matrix Analysis for Statistics. New York: Wiley, 1980.

[25] J. P. Delmas, "Asymptotically minimum variance second-order estimation for non-circular signals with application to DOA estimation," IEEE Trans. Signal Process., vol. 52, no. 5, pp. 1235-1241, May 2004

[26] F. Roemer and M. Haardt, "Using 3-D unitary ESPRIT on a hexagonal shaped ESPAR antenna for 1-D and 2-D DOA estimation," presented at the IEEE ITG Workshop on Smart Antennas, Duisburg, Germany, Apr. 2005.

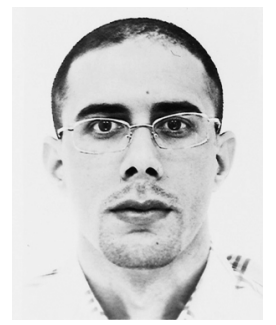

Habti Abeida was born in Settat, Morocco, on October 20, 1977. He received the B.S. degrees in applied mathematics from Hassan II University, Casablanca, Morocco, and René Descartes University, Paris, France in 2000 and 2001, respectively, and the Master's degree in statistics and Ph.D. degree in applied mathematics both from Pierre et Marie Curie University, Paris, France, in 2002 and 2006, respectively.

$\mathrm{He}$ is currently a Postdoctoral Researcher at the Department of Telecommunication, Supelec, Gif-sur-Yvette, France. His research interests are in the areas of statistical signal processing with application to communications and antenna array.

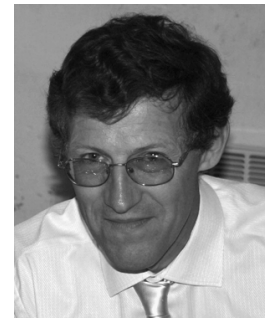

Jean-Pierre Delmas (M'00-SM'06) was born in France in 1950. He received the Engineering degree from Ecole Centrale de Lyon, France, in 1973 , the Certificat d'Études Supérieures from the Ecole Nationale Supérieure des Télécommunications, Paris, France, in 1982, and the Habilitation à Diriger des Recherches (HDR) degree from the University of Paris, Orsay, France, in 2001.

Since 1980, he has been with the Institu TELECOM SudParis, where he is presently a Professor in the CITI department and Deputy Director of the UMR CNRS 5157 (SAMOVAR) laboratory. His teaching and research interests are in the areas of statistical signal processing with application to communications and antenna array.

Dr. Delmas has served as Associate Editor with the IEEE TRANSACTIONS ON SIGNAL PROCESSING from 2002 to 2006. 